\title{
Non-LTE chemical abundances in Galactic open and globular clusters $\star, \star \star$
}

\author{
Mikhail Kovalev ${ }^{1}$, Maria Bergemann ${ }^{1}$, Yuan-Sen Ting ${ }^{2,3,4, \star \star \star}$, and Hans-Walter Rix ${ }^{1}$ \\ ${ }^{1}$ Max Planck Institute for Astronomy, Königstuhl 17, 69117 Heidelberg, Germany \\ e-mail: kovalev@mpia.de \\ 2 Institute for Advanced Study, Princeton, NJ 08540, USA \\ 3 Department of Astrophysical Sciences, Princeton University, Princeton, NJ 08544, USA \\ ${ }^{4}$ Observatories of the Carnegie Institution of Washington, 813 Santa Barbara Street, Pasadena, CA 91101, USA
}

Received 9 May 2019 / Accepted 1 July 2019

\begin{abstract}
Aims. We study the effects of non-local thermodynamic equilibrium (NLTE) on the determination of stellar parameters and abundances of $\mathrm{Fe}, \mathrm{Mg}$, and Ti from the medium-resolution spectra of FGK stars.

Methods. We extended the Payne fitting approach to draw on NLTE and LTE spectral models. These were used to analyse the spectra of the Gaia-ESO benchmark stars and the spectra of 742 stars in 13 open and globular clusters in the Milky Way: NGC 3532, NGC 5927, NGC 2243, NGC 104, NGC 1851, NGC 2808, NGC 362, M 2, NGC 6752, NGC 1904, NGC 4833, NGC 4372, and M15. Results. Our approach accurately recovers effective temperatures, surface gravities, and abundances of the benchmark stars and clusters members. The differences between NLTE and LTE are significant in the metal-poor regime, $[\mathrm{Fe} / \mathrm{H}] \lesssim-1$. The NLTE $[\mathrm{Fe} / \mathrm{H}]$ values are systematically higher, whereas the average NLTE $[\mathrm{Mg} / \mathrm{Fe}]$ abundance ratios are $\sim 0.15$ dex lower, compared to LTE. Our LTE measurements of metallicities and abundances of stars in Galactic clusters are in a good agreement with the literature. Though, for most clusters, our study yields the first estimates of NLTE abundances of Fe, $\mathrm{Mg}$, and Ti.

Conclusion. All clusters investigated in this work are homogeneous in $\mathrm{Fe}$ and $\mathrm{Ti}$, with the intra-cluster abundance variations of less then 0.04 dex. NGC 2808, NGC 4833, M 2, and M 15 show significant dispersions in $[\mathrm{Mg} / \mathrm{Fe}]$. Contrary to common assumptions, the NLTE analysis changes the mean abundance ratios in the clusters, but it does not influence the intra-cluster abundance dispersions.
\end{abstract}

Key words. stars: abundances - stars: fundamental parameters - techniques: spectroscopic - open clusters and associations: general globular clusters: general

\section{Introduction}

Fast and reliable modelling of stellar spectra is becoming increasingly important for current stellar and Galactic astrophysics. Large-scale spectroscopic stellar surveys, such as GaiaESO (Gilmore et al. 2012; Randich et al. 2013), APOGEE (Majewski et al. 2017), and GALAH (De Silva et al. 2015) are revolutionising our understanding of the structure and evolution of the Milky Way galaxy, stellar populations, and stellar physics. The ever increasing amount of high-quality spectra, in return, demands rigorous, physically realistic, and efficient data analysis techniques to provide an accurate diagnostic of stellar parameters and abundances. This problem has two sides. Precise spectral fitting and analysis requires powerful numerical optimisation and data-model comparison algorithms. On the other hand, the accuracy of stellar label estimates is mostly limited by the physics of spectral models used in the model-data comparison. The fitting aspect has been the subject of extensive studies over the past years, and various methods (e.g. Recio-Blanco et al. 2006; Schönrich \& Bergemann 2014;

* Full Table A.5 is only available at the CDS via anonymous ftp to cdsarc.u-strasbg. fr (130.79.128.5) or via http://cdsarc. u-strasbg.fr/viz-bin/qcat?]/A+A/628/A54

$\star \star$ Based on data products from observations made with ESO Telescopes at the La Silla Paranal Observatory under programme ID 188. B-3002.

$\star \star \star$ Hubble Fellow.
Ness et al. 2015; Casey et al. 2017; Ting et al. 2019) have been developed and applied to the analysis of large survey datasets.

Major developments have also occurred in the field of stellar atmosphere physics. Non-local thermodynamic equilibrium (NLTE) radiative transfer is now routinely performed for many elements in the periodic table. This allows detailed calculations of spectral profiles that account for NLTE effects. NLTE models consistently describe the interaction of the gas particles in stellar atmospheres with the radiation field (Auer \& Mihalas 1969), in this respect being more realistic than LTE models. In NLTE, photons affect atomic energy level populations, whilst in LTE those are set solely by the Saha equation for ionisation and by the Boltzmann distribution for excitation. NLTE models predict more realistic absorption line profiles and hence provide more accurate stellar parameters and abundances (e.g. Ruchti et al. 2013; Zhao et al. 2016). However NLTE models are often incomplete in terms of atomic data, such as collisions with $\mathrm{H}$ atoms and electrons or photo-ionisation crosssections. Major efforts to improve atomic data are underway (e.g. Yakovleva et al. 2016; Bautista et al. 2017; Belyaev \& Yakovleva 2017; Barklem et al. 2017; Amarsi et al. 2018; Barklem 2018) and there is no doubt that many gaps in the existing atomic and molecular databases will be filled in the near-term future. Besides, strictly speaking, no single NLTE model is complete in terms of atomic data, and also quantum-mechanical crosssections are usually available for a small part of the full atomic or molecular system (Barklem 2016). 
In this work, we study the effect of NLTE on the analysis of stellar parameters and chemical abundances for FGK-type stars. We combined NLTE stellar spectral models with the Payne ${ }^{1}$ code developed by Ting et al. (2019) and applied our methods to the observed stellar spectra from the third public data release by the Gaia-ESO survey. This work is a proof of concept of the combined NLTE-Payne approach and it is, hence, limited to the analysis to the Gaia-ESO benchmark stars and a sample of Galactic open and globular clusters, for which independent estimates of stellar labels (both stellar parameters and detailed abundances) are available from earlier studies.

The paper is organised as follows. In Sect. 2, we describe the observed sample, the physical aspects of the theoretical spectral models, and the mathematical basis of the Payne code. We present the LTE and NLTE results in Sect. 3 and compare them with the literature in Sect. 4. Section 5 summarises the conclusions and outlines future prospects arising from this work.

\section{Methods}

\subsection{Observed spectra}

We use the spectra of FGK stars observed within the GaiaESO spectroscopic survey (Gilmore et al. 2012; Randich et al. 2013). These spectra are now publicly available as a part of the third data release (DR3.1) ${ }^{2}$. The data were obtained with the Giraffe instrument (Pasquini et al. 2002) at the ESO (European Southern Observatory) VLT (Very Large Telescope). We use the spectra taken with the HR10 setting, which covers $280 \AA$ from $5334 \AA$ to $5611 \AA$, at a resolving power of $R=\lambda / \Delta \lambda \sim 19800$. The average signal-to-noise ratio $(\mathrm{S} / \mathrm{N})$ of a spectrum ranges from 90 to 2800 per $\AA^{3}$, with the majority of the spectra sampling the $\mathrm{S} / \mathrm{N}$ in range of $150-200 \AA^{-1}$.

Our observed sample contains 916 FGK-type stars with luminosity classes from III to $\mathrm{V}$ that includes main-sequence (MS), subgiants, and red giant branch (RGB) stars. A fraction of these are the Gaia-ESO benchmark stars (174 spectra of 19 stars), but we also include 742 stars in two open and 11 globular clusters. We exclude four benchmark stars with effective temperature $T_{\text {eff }}<4000 \mathrm{~K}$, because this regime of stellar parameters is not covered by our model atmosphere grids. $\beta$ Ara is not a part of our calibration sample, as it is not recommended as a benchmark in Pancino et al. (2017a). These stars were previously analysed by Gaia-ESO (Smiljanic et al. 2014; San Roman et al. 2015; Pancino et al. 2017b) and are included in the Gaia-ESO DR3 catalogue.

We estimate the radial velocity (RV) by cross-correlating the observed spectrum with a synthetic, metal-poor spectral template $\left(T_{\text {eff }}=5800 \mathrm{~K}, \log (g)=4.5 \mathrm{dex},[\mathrm{Fe} / \mathrm{H}]=-2 \mathrm{dex}\right)^{4}$, which is shifted in the RV range of $\pm 400 \mathrm{~km} \mathrm{~s}^{-1}$ with a step of $0.5 \mathrm{~km} \mathrm{~s}^{-1}$. We computed the cross-correlation function for all RV values and fitted a parabola to 20 points around the maximum value of the cross-correlation function. Then we applied the Doppler-shift to the observed spectrum using the velocity value at the position of the peak of the parabola. Since

\footnotetext{
1 https://github.com/tingyuansen/The_Payne

2 http://archive.eso.org/wdb/wdb/adp/phase3_spectral/ form?collection_name=GAIAESO

3 We employ the following relationship: $S / N\left[\AA^{-1}\right]=\sqrt{20} S / N$ [pixel ${ }^{-1}$ ], where 20 pixels are equivalent to $1 \AA$, that is, the sampling of the Giraffe HR10 spectra.

4 Our tests showed that this template provides robust RV estimates for the full metallicity range.
}

cross-correlation can incur small errors due to step size or template choice, we later fitted for residual shift in the range of $\pm 2 \mathrm{~km} \mathrm{~s}^{-1}$.

\subsection{Model atmospheres and synthetic spectra}

The grids of LTE and NLTE synthetic spectra were computed using the new online spectrum synthesis tool ${ }^{5}$. The model atmospheres are 1D plane-parallel hydrostatic LTE models taken from the MAFAGS-OS grid (Grupp 2004a,b). For the NLTE grid we first compute the NLTE atomic number densities for Mg (Bergemann et al. 2017a), Ti (Bergemann 2011), Fe (Bergemann et al. 2012), and Mn (Bergemann \& Gehren 2008) using the DETAIL statistical equilibrium (SE) code (Butler \& Giddings 1985). These were then fed into the SIU (Reetz 1991) radiative transfer (RT) and spectrum synthesis code. In total, 626 spectral lines of Mg I, Ti I, Fe I and Mn I are modelled in NLTE for the NLTE grid, while for the LTE grid these lines are modelled with default LTE atomic level populations. Our approach is conceptually similar to Buder et al. (2019), but we employed different SE and RT codes. We chose to use the MAFAGS-OS atmosphere grids, because these are internally consistent with DETAIL and SIU. In particular, the latter codes adopt the atomic and molecular partial pressures and partition functions that are supplied with the MAFAGS-OS models.

We computed 20000 spectral models with $T_{\text {eff }}$ uniformly distributed in the range from 4000 to $7000 \mathrm{~K}$ and $\log (g)$ s in the range from 1.0 to 5.0 dex. Metallicity ${ }^{6},[\mathrm{Fe} / \mathrm{H}]$, is uniformly distributed in the range from $[\mathrm{Fe} / \mathrm{H}]=-2.6$ to $0.5 \mathrm{dex}$. We also allowed for random variations in the rations of the magnesium, titanium, manganese to iron: $[\mathrm{Mg} / \mathrm{Fe}],[\mathrm{Ti} / \mathrm{Fe}]$ from -0.4 to $0.8 \mathrm{dex}$ and $[\mathrm{Mn} / \mathrm{Fe}]$ from -0.8 to 0.4 dex. The abundances of other chemical elements were assumed to be solar and follow the iron abundance $[\mathrm{Fe} / \mathrm{H}]$. In the metal-poor regime $([\mathrm{Fe} / \mathrm{H}]<$ $-1 \mathrm{dex}$ ), some elements (like important opacity contributors $\mathrm{C}$ and $\mathrm{O})$ can be significantly enhanced relative to the solar values. Therefore we computed several metal-poor synthetic spectra using a 0.5 dex enhancement of $\mathrm{C}$ and $\mathrm{O}$ abundances and found that there is no impact on the spectral models. Micro-turbulence varies from 0.6 to $2.0 \mathrm{~km} \mathrm{~s}^{-1}$, in line with high-resolution studies of FGK stars (e.g. Ruchti et al. 2013). The detailed solar abundances assumed in the MAFAGS-OS grids were reported in Grupp (2004a). For the elements treated in NLTE, we adopted $\log A(\mathrm{Mg})_{\odot}=7.58 \mathrm{dex}, \log A(\mathrm{Ti})_{\odot}=4.94 \mathrm{dex}, \log A(\mathrm{Mn})_{\odot}=$ $5.53 \mathrm{dex}$ and $\log A(\mathrm{Fe})_{\odot}=7.50 \mathrm{dex}$ (meteoritic values from Grevesse \& Sauval 1998).

The widths of spectral lines in the observed spectra depend on many effects, such as the properties of the instrument, turbulence in stellar atmospheres, and stellar rotation (Gray 2005). However, it is not possible to separate these effects at the resolution of the Giraffe spectra. Hence, the macroturbulence, $V_{\text {mac }}$, and the projected rotation velocity, $V \sin i$, were dealt with by smoothing the model spectra with a Gaussian kernel, which corresponds to a characteristic velocity $V_{\text {broad }}$ in the range from 5.0 to $25.0 \mathrm{~km} \mathrm{~s}^{-1}$ that encompasses the typical values of $V_{\mathrm{mac}}$ and $V \sin i$ reported for FGK stars (Gray 2005; Jofré et al. 2015). After that, the synthetic spectra were degraded to the resolution of the HR10 setup by convolving them with an instrumental profile (Appendix B) and were re-sampled onto the observed spectrum wavelength grid using the sampling of 20 wavelength points per $\AA$.

\footnotetext{
5 http://nlte.mpia.de

6 Hereafter, the abundance of iron $[\mathrm{Fe} / \mathrm{H}]$, is used as a proxy for metallicity.
} 


\subsection{The Payne code}

The data-model comparison was not performed directly. Instead, we used the Payne code to interpolate in the grid of synthetic spectra. The approach consists of two stages: the training (model building) and the test (data fitting) steps. In the training step, we built a Payne model using a set of pre-computed LTE and NLTE stellar spectra. We approximate the variation of the flux using an artificial neural network (ANN). In the test step, $\chi^{2}$ minimisation was employed to find the best-fit stellar parameters and abundances by comparing the model spectra to the observations. In what follows, we describe the key details of the method. For more details on the algorithm, we refer the reader to Ting et al. (2019).

The conceptual idea of the code is simple. We employed a simple ANN that consists of several fully connected layers of neurons: an input layer, two hidden layers, and an output layer. The input data are given by a set of stellar parameters (hereafter, labels) $T_{\text {eff }}, \log (g), V_{\text {mic }}, V_{\text {broad }},[\mathrm{Fe} / \mathrm{H}],[\mathrm{Mg} / \mathrm{Fe}],[\mathrm{Ti} / \mathrm{Fe}]$, and $[\mathrm{Mn} / \mathrm{Fe}]$. The output data comprise the normalised flux values tabulated on a wavelength grid, as a function of the input labels.

Three hundred neurons in each hidden layer apply a weight and an offset to the output from the previous layer, and these outputs are activated using a $\operatorname{ReLU}(z)=\max (z, 0)$ function for the first layer and a sigmoid function $s(z)=\left(1+e^{-z}\right)^{-1}$ for the second layer. A subset of the precomputed spectral grid (that is 15000 synthetic spectra) was used to train the ANN, whereby the weights and the offsets are adjusted to the optimal values. This subset is referred to as a training set. We trained the neural networks by minimising the $L^{2}$ loss. In other words, we computed a minimal sum of the Euclidean distances between the target ab-initio flux from the training set and the flux predicted by the model at each wavelength point. We used crossvalidation with the remaining set of 5000 spectra, which were referred to as a cross-validation set to prevent over-fitting. This requires optimal values of the ANN to decrease $L^{2}$ loss also for the cross-validation set, which is not directly used during training. Together, the ANN layers act like a function that predicts a flux spectrum for a set of given labels. The main difference of the current implementation of Payne with respect to the one in Ting et al. (2019) is that we used only one ANN to represent the full stellar spectrum. In our realisation ${ }^{7}$ an ANN can exploit information from the adjacent pixels, while previously each individual pixel was trained separately. A synthetic spectrum was generated at arbitrary points in stellar parameter space within the domain of the training grid and was compared to the observed spectrum. A standard $\chi^{2}$ minimisation was used to compute the likelihood of the fit and, hence, to find the stellar parameters that best characterise the observed spectrum. We also allowed for a small Doppler shift, $\pm 2 \mathrm{~km} \mathrm{~s}^{-1}$, on top of the RV from crosscorrelation, to optimise the spectral fit.

The continuum normalisation of the observed spectra was performed during the $\chi^{2}$ minimisation. We searched for the coefficients of a linear combination of the first ten Chebyshev polynomials, which represents a function that fits the shape of the continuum, using the full observed spectrum. A synthetic spectrum was then multiplied with this function.

In total, for each observed spectrum, we optimised 19 free parameters: one Doppler shift, eight spectral labels and ten coefficients of Chebyshev polynomials. The abundances of individual elements were derived simultaneously with other stellar parameters via the full spectral fitting process. We also employed

The most recent version on Github: https://github.com/ tingyuansen/The_Payne the classical method of fitting separately each spectral line using line masks. However, this method delivered less precise abundances, as gauged by the star-to-star scatter, hence, we did not use the line masks in the final abundance analysis.

Following the result in Bergemann (2011) which strongly recommended to use only Ti II lines in abundance analysis, we masked out all Ti I lines. We note, however, that we did not include NLTE calculations for Ti II, as the NLTE effects on this ion are very small in the metallicity regime of our sample (Bergemann 2011). Hence, the difference between our LTE and NLTE Ti abundance reflects only an indirect effect of NLTE on stellar parameters.

\subsection{Internal accuracy of the method}

We verified the internal accuracy of the method by subjecting it to tests similar to those employed by Ting et al. (2019). First, we compared the interpolated synthetic spectra to the original models from the cross-validation sample. In this case we explore how well the Payne can generate new spectrum. The median interpolation error of the flux across 5000 models is $\leq 10^{-3}$, that is, within 0.1 .

Second, we test how well we can recover original labels from the model, through $\chi^{2}$ minimisation. In this case we apply random Doppler shift, multiply the model spectrum by a random combination of the first ten Chebyshev polynomials, that represent the continuum level and add noise. Such a modified model served as a fair representation of a real observed spectrum. The tests were performed for the noiseless models and the models degraded to a S/N of $90 \AA^{-1}$ and $224 \AA^{-1}$. This range of $\mathrm{S} / \mathrm{N}$ brackets the typical values of the observed HR10 spectra, with the majority of the spectra sampling the $\mathrm{S} / \mathrm{N}$ range of 150 $200 \AA^{-1}$. The typical S/N of the spectra of the benchmark stars is $\sim 200 \AA^{-1}$.

Table 1 presents the average differences between the input and the output stellar parameters for the cross-validation sample. The scatter is represented by one standard deviation. To facilitate the analysis, we grouped the results into three metallicity bins.

The results for the noiseless models with $[\mathrm{Fe} / \mathrm{H}]$ in the range from -1.6 to 0.5 dex suggest high internal accuracy of the method. For the lower-metallicity models, there is a small bias and a larger dispersion in the residuals, because we have less spectral information in this regime. The bias is also marginal for the high-S/N spectra with $S / N=224 \AA^{-1}$, although the scatter in the output is increased compared to the noiseless models. Our analysis of the noisy models, $S / N=90 \AA^{-1}$, yields acceptable results for the metal-rich and moderately metal-poor stars with $[\mathrm{Fe} / \mathrm{H}] \gtrsim$ -1.6 dex. On the other hand, the most metal-poor noisy spectra are not fitted well. Despite a modest bias in $T_{\text {eff }}$, the dispersion of $\log g$ and the abundance ratios is very large and may require a different approach to obtain high-precision abundances in this regime. According to this test, good Mn abundances (better than $\sim 0.1$ dex) can be derived only for metal-rich stars.

These tests illustrate only the internal accuracy of the Payne model reconstruction and, hence, set the minimum uncertainty on the parameters determined by our method, regardless of the training sample, its physical properties and completeness. The analysis of observed data may result in a larger uncertainty, as various other effects, such as the physical complexity of the model atmospheres and synthetic spectra and properties of the observed data (data reduction effects etc.), will contribute to the total uncertainties. We tested this in the next section by analysing the Gaia-ESO benchmark stars. 
Table 1. Internal errors of label's recovery by the Payne see Sect. 2.4 for details.

\begin{tabular}{|c|c|c|c|c|c|c|c|c|c|}
\hline $\begin{array}{l}S / N \\
\AA^{-1} \\
\end{array}$ & $\begin{array}{c}{[\mathrm{Fe} / \mathrm{H}]} \\
\operatorname{dex}\end{array}$ & $\begin{array}{c}\Delta T_{\text {eff }} \\
1000 \mathrm{~K}\end{array}$ & $\begin{array}{c}\Delta \log (g) \\
\operatorname{dex}\end{array}$ & $\begin{array}{c}\Delta V_{\text {mic }} \\
\mathrm{km} \mathrm{s}^{-1}\end{array}$ & $\begin{array}{c}\Delta V_{\text {broad }} \\
10 \mathrm{~km} \mathrm{~s}^{-1}\end{array}$ & $\begin{array}{c}\Delta[\mathrm{Fe} / \mathrm{H}] \\
\operatorname{dex}\end{array}$ & $\begin{array}{c}\Delta[\mathrm{Mg} / \mathrm{Fe}] \\
\operatorname{dex}\end{array}$ & $\begin{array}{c}\Delta[\mathrm{Ti} / \mathrm{Fe}] \\
\operatorname{dex}\end{array}$ & $\begin{array}{c}\Delta[\mathrm{Mn} / \mathrm{Fe}] \\
\operatorname{dex}\end{array}$ \\
\hline \multirow[t]{3}{*}{90} & $-2.6:-1.6$ & $0.00 \pm 0.27$ & $-0.05 \pm 0.56$ & $-0.10 \pm 0.77$ & $-0.01 \pm 0.29$ & $-0.00 \pm 0.18$ & $0.01 \pm 0.17$ & $-0.02 \pm 0.34$ & $-0.01 \pm 0.64$ \\
\hline & $-1.6:-0.6$ & $0.01 \pm 0.12$ & $0.00 \pm 0.21$ & $0.01 \pm 0.26$ & $-0.00 \pm 0.09$ & $0.01 \pm 0.07$ & $0.00 \pm 0.10$ & $-0.01 \pm 0.13$ & $-0.01 \pm 0.37$ \\
\hline & $-0.6: 0.5$ & $0.01 \pm 0.07$ & $0.00 \pm 0.12$ & $0.00 \pm 0.10$ & $-0.00 \pm 0.05$ & $0.01 \pm 0.06$ & $-0.00 \pm 0.09$ & $-0.00 \pm 0.07$ & $-0.00 \pm 0.11$ \\
\hline \multirow[t]{3}{*}{224} & $-2.6:-1.6$ & $-0.00 \pm 0.11$ & $-0.04 \pm 0.25$ & $-0.00 \pm 0.41$ & $0.01 \pm 0.13$ & $-0.00 \pm 0.07$ & $0.01 \pm 0.07$ & $-0.01 \pm 0.17$ & $-0.03 \pm 0.55$ \\
\hline & $-1.6:-0.6$ & $-0.00 \pm 0.05$ & $-0.00 \pm 0.08$ & $-0.00 \pm 0.09$ & $-0.00 \pm 0.04$ & $-0.00 \pm 0.03$ & $0.00 \pm 0.05$ & $-0.00 \pm 0.05$ & $-0.03 \pm 0.25$ \\
\hline & $-0.6: 0.5$ & $0.00 \pm 0.03$ & $0.00 \pm 0.06$ & $0.00 \pm 0.05$ & $-0.00 \pm 0.02$ & $0.00 \pm 0.04$ & $0.00 \pm 0.04$ & $0.00 \pm 0.03$ & $0.00 \pm 0.05$ \\
\hline No & $-2.6:-1.6$ & $-0.00 \pm 0.02$ & $-0.01 \pm 0.06$ & $-0.01 \pm 0.15$ & $0.00 \pm 0.02$ & $-0.00 \pm 0.02$ & $-0.00 \pm 0.03$ & $-0.00 \pm 0.04$ & $-0.01 \pm 0.21$ \\
\hline \multirow[t]{2}{*}{ noise } & $-1.6:-0.6$ & $-0.00 \pm 0.01$ & $0.00 \pm 0.03$ & $0.00 \pm 0.03$ & $0.00 \pm 0.01$ & $0.00 \pm 0.01$ & $-0.00 \pm 0.02$ & $0.00 \pm 0.02$ & $0.00 \pm 0.05$ \\
\hline & $-0.6: 0.5$ & $0.00 \pm 0.01$ & $0.00 \pm 0.05$ & $0.00 \pm 0.03$ & $0.00 \pm 0.01$ & $0.00 \pm 0.05$ & $-0.00 \pm 0.04$ & $0.00 \pm 0.01$ & $0.00 \pm 0.03$ \\
\hline
\end{tabular}

\section{Results}

\subsection{Gaia-ESO benchmark stars}

Our results for the Gaia-ESO benchmark stars are shown in Figs. 1 and 2. Figure 1 compares our NLTE stellar parameters with the values from Jofré et al. (2015), Schönrich \& Bergemann (2014), and with the Gaia-ESO DR3 catalogue (GES; Smiljanic et al. 2014). In Jofré et al. (2015), $T_{\text {eff }}$ estimates were determined from photometry and interferometry, $\log (g)$ from parallaxes and astroseismology. $[\mathrm{Fe} / \mathrm{H}]$ estimates were obtained from the NLTE analysis of Fe lines in the high-resolution spectra taken with the UVES, NARVAL and HARPS spectrographs (Blanco-Cuaresma et al. 2014). In order to be consistent with our reference solar $[\mathrm{Fe} / \mathrm{H}]$ scale, we subtracted 0.05 dex from Jofre and GES metallicities, as they are based on the Grevesse et al. (2007) metallicity scale $\left(\log A(\mathrm{Fe})_{\odot}=7.45 \mathrm{dex}\right)$. Likewise, we subtracted 0.03 dex from Amarsi et al. (2016) metallicities, as they employ $\log A(\mathrm{Fe})_{\odot}=7.47 \mathrm{dex}$.

The estimates of stellar parameters in Schönrich \& Bergemann (2014) are derived using a full Bayesian approach by solving for the posterior in a multi-dimensional parameter space, including photometry, high-resolution spectra, parallaxes, and evolutionary constraints. The estimates of stellar parameters in the Gaia-ESO DR3 catalogue rely on the highresolution (UVES at VLT) spectroscopy only.

Figure 1 suggests that the agreement of our NLTE results with the literature studies is very good. The differences with Jofré et al. (2015) are of the order $-29 \pm 88 \mathrm{~K}$ in $T_{\text {eff }}, 0.09 \pm 0.16$ dex in $\log (g)$ and $0.02 \pm 0.09 \mathrm{dex}$ in $[\mathrm{Fe} / \mathrm{H}]$ across the full parameter space, and they also compare favourably with the results obtained by Schönrich \& Bergemann (2014) and reported in the Gaia-ESO DR3 catalogue. Results for individual stars are listed in Table A.2. The scatter is slightly larger for the metal-poor stars. This could be the consequence of the limited coverage of the training set. In particular, the two very metal-poor evolved stars HD 122563 and HD 140283 are located next to the low-metallicity edge of our training grid. Since the Gaia-ESO benchmark star sample contains only three stars with $[\mathrm{Fe} / \mathrm{H}]<-1$, no reliable statistics can be drawn on the success of our approach in this regime of stellar parameters. Also the sample of RGB stars is very small and contains only five objects with $\log (g)<3$ dex. We address the performance of our method for low-gravity stars in the next section, by analysing a set of open and globular clusters that cover a large metallicity range, $-2.3 \lesssim[\mathrm{Fe} / \mathrm{H}] \lesssim-0.1 \mathrm{dex}$, and provide a better sampling on the RGB.

Figure 2 illustrates the performance of our method for the spectra taken at different exposure times. We have chosen four stars representative of our calibration sample: HD 107328 - a moderately metal-poor giant $\left(T_{\text {eff }}=4384 \mathrm{~K}, \log (g)=1.90 \mathrm{dex}\right.$, and $\left.[\mathrm{Fe} / \mathrm{H}]_{\mathrm{NLTE}}=-0.60 \mathrm{dex}\right), \xi$ Hya - a metal-rich subgiant $\left(T_{\mathrm{eff}}=5045 \mathrm{~K}, \log (g)=3.01 \mathrm{dex}\right.$, and $[\mathrm{Fe} / \mathrm{H}]_{\mathrm{NLTE}}=-0.05$ dex $), \epsilon$ For - a moderately metal-poor subgiant $\left(T_{\text {eff }}=5070 \mathrm{~K}\right.$, $\log (g)=3.28 \mathrm{dex}$, and $\left.[\mathrm{Fe} / \mathrm{H}]_{\mathrm{NLTE}}=-0.65 \mathrm{dex}\right)$, and $\alpha \mathrm{Cen} B$ - a metal-rich dwarf $\left(T_{\text {eff }}=5167 \mathrm{~K}, \log (g)=4.33 \mathrm{dex}\right.$, and $\left.[\mathrm{Fe} / \mathrm{H}]_{\mathrm{NLTE}}=0.14 \mathrm{dex}\right)$. These stars have been observed with different exposure times, corresponding to the S/Ns of 100$2500 \AA^{-1}$ that allows us to validate the differential precision of the adopted model. We do not detect any evidence of a systematic bias that depends on the data quality. In particular, the mean difference (taken as one standard deviation) between abundances of Fe, $\mathrm{Mg}$, and Ti obtained from the $S / N \sim 100 \AA^{-1}$ spectra and those obtained from the highest-quality data $\left(S / N \sim 2000 \AA^{-1}\right)$ is not larger than 0.02 dex for any of these stars, and is less than 0.01 dex for the majority. We hence conclude that our results are not very sensitive to the quality of the observed data for a wide range of $\mathrm{S} / \mathrm{Ns}$.

\subsection{Open and globular clusters}

\subsubsection{Sample selection}

Our dataset includes two open clusters and 11 globular clusters. The cluster members are chosen using the central coordinates and the RV estimates from the $\mathrm{SIMBAD}^{8}$ database listed in Table A.3. We select only stars with an RV within $5 \mathrm{~km} \mathrm{~s}^{-1}$ from the cluster median ${ }^{9}$, for the open clusters. For the globular clusters, we assume a $1 \sigma \mathrm{RV}$ dispersion and the central values from Pancino et al. (2017a). We also apply a $2 \sigma$ clipping around the median in metallicity, and employ proper motions from Gaia DR2 (Gaia Collaboration 2018) to exclude stars outside the $2 \sigma$ range from the median proper motion of each cluster. It is common to use distances to compute astrometric gravities (e.g. Ruchti et al. 2013). However, the majority of clusters in our sample are located at heliocentric distances $d_{\odot}$ of $>2 \mathrm{kpc}$, where parallaxes are very uncertain. Besides, poorly constrained differential extinction in some clusters limits the applicability of standard relations, to derive $\log (g)$ from distances and photometric magnitudes. We, hence, refrain from using the GDR2 parallaxes to compute surface gravities. Instead, we compare our results with the isochrones computed using our estimates of metallicities and the ages adopted from literature studies, in particular, from Kruijssen et al. (2019) for GCs and from the

\footnotetext{
8 http://simbad.u-strasbg.fr/simbad/

9 The median is used because it is less sensitive to outliers.
} 

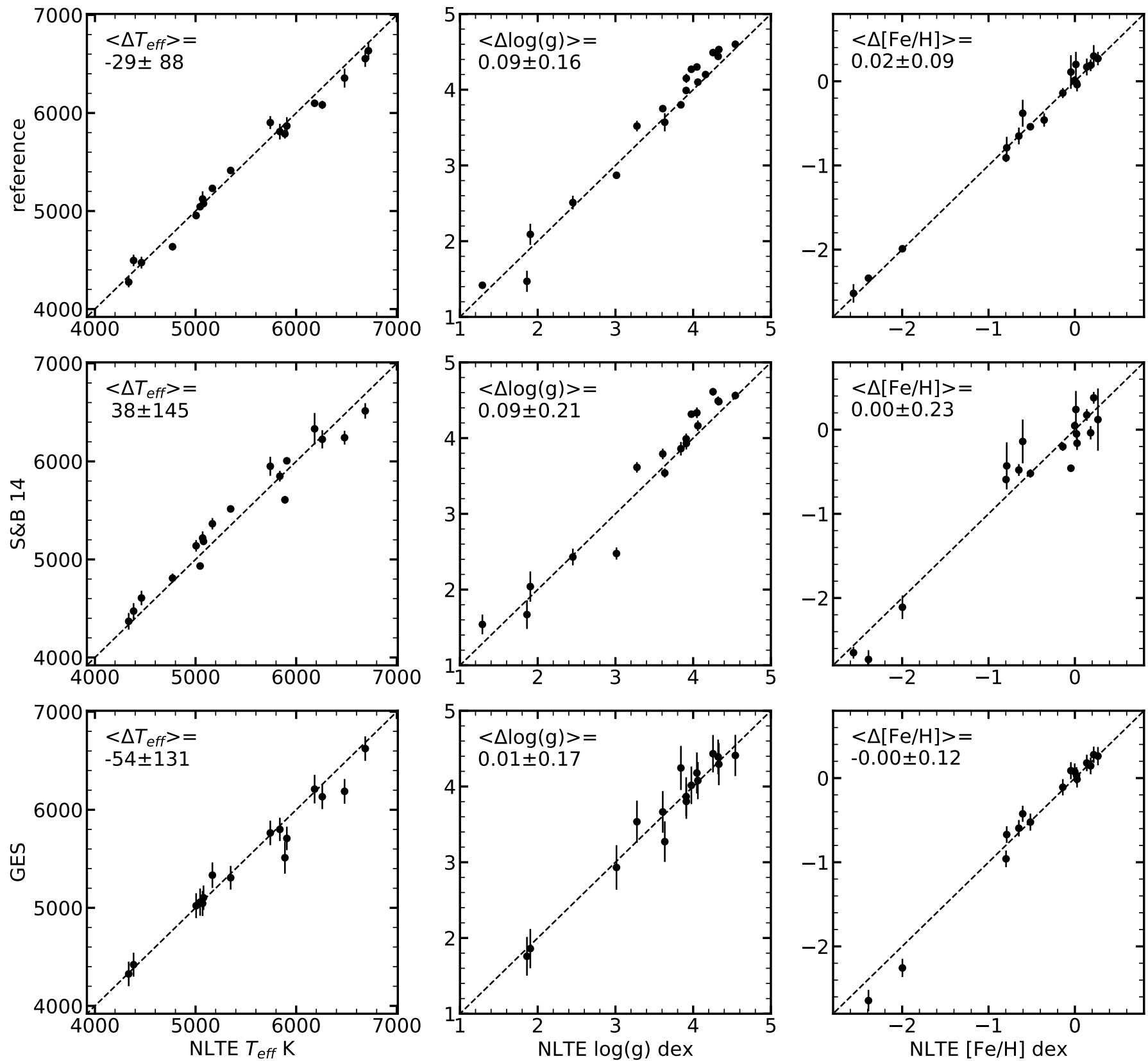

Fig. 1. NLTE spectroscopic estimates for benchmark stars compared with literature. Top panels: reference stellar parameters and their uncertainties from Jofré et al. (2015), Karovicova et al. (2018), Amarsi et al. (2016). Middle and bottom panels: our values against the results from Schönrich \& Bergemann (2014) and the GES catalogue Smiljanic et al. (2014), respectively. The mean offset and scatter are given in the legend of each plot.

WEBDA database ${ }^{10}$ for open clusters. For most clusters, the ages are derived from the colour-magnitude diagram turn-off (TO) or horizontal branch (HB) fits. Hence, also this comparison can be performed only with the caveat that the TO or HB ages are not a fundamental reference, but are model-dependent and may not be fully unbiased.

\subsubsection{Stellar parameters and comparison with the isochrones}

The majority of the globular clusters are distant and are represented by RGB stars in our sample. Main-sequence stars are observed only in the nearby metal-rich open cluster NGC 3532.

\footnotetext{
${ }^{10}$ https://www . univie.ac .at/webda/
}

Hence, in what follows, the discussion will mainly focus on the RGB population across a wide range of metallicities, from -0.5 (NGC 5927) to -2.3 dex (M 15).

In Fig. 3, we compare NLTE and LTE stellar parameters as a function of NLTE metallicity. Since most stars, within a cluster, are in the same evolutionary stage (lower or upper RGB), we have chosen to show only the mean NLTE-LTE differences, averaged over all stars in a given cluster. This is sufficient to illustrate the key result: the differences between NLTE and LTE measurements of $T_{\text {eff }}, \log g$ and $[\mathrm{Fe} / \mathrm{H}]$ vary in lockstep with metallicity. This reflects the NLTE effects in the formation of the Fe I and Ti I spectral lines, which are ubiquitous in HR10. It is furthermore important, although not unexpected, that below $[\mathrm{Fe} / \mathrm{H}] \sim-1$ dex the changes are nearly linear, consistent with our earlier theoretical estimates (Lind et al. 2012) and with the 


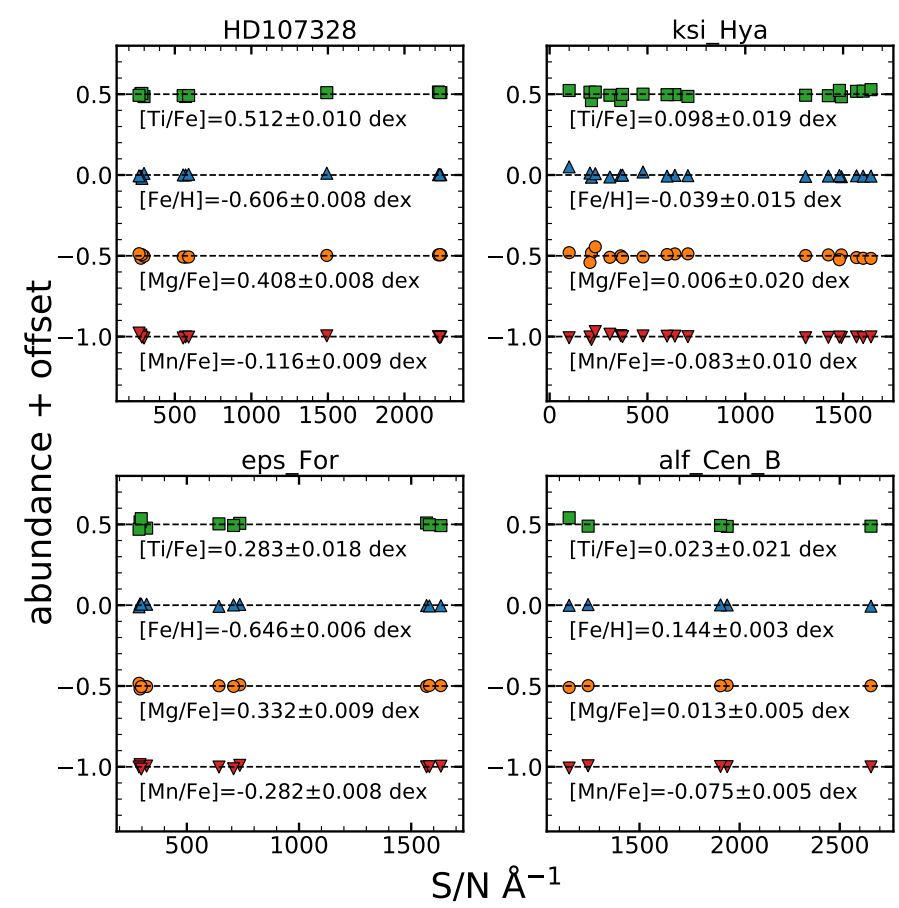

Fig. 2. NLTE elemental abundances derived from the spectra taken at different exposure times. Abundances determined at $S / N=200 \AA^{-1}$ are just as precise as those at $S / N>2000 \AA^{-1}$, see Sect. 3.1 for details.

analysis of the metal-poor field stars in the Milky Way (Ruchti et al. 2013). The NLTE effect is most striking at $[\mathrm{Fe} / \mathrm{H}] \lesssim-2$, where we find differences of $\sim 300 \mathrm{~K}$ in $T_{\text {eff }}, \sim 0.6$ dex in $\log g$ and $\sim 0.3$ dex in $[\mathrm{Fe} / \mathrm{H}]$. The $[\mathrm{Mg} / \mathrm{Fe}]$ ratios tend to be lower in NLTE, this reflects negative NLTE abundance corrections for the only Mg line in HR10 (Mg I 5528 $\AA$ ), which is consistent with earlier studies (Osorio et al. 2015; Bergemann et al. 2017b). The upturn in $[\mathrm{Mg} / \mathrm{Fe}]$ at $[\mathrm{Fe} / \mathrm{H}] \sim-2$ dex is real and it is caused by the change of the dominant NLTE effect at this metallicity. At higher $[\mathrm{Fe} / \mathrm{H}]$, strong line scattering and photon loss, and, hence, the deviations of the source function from the Planck function, play an important role in the statistical equilibrium of the ion. However, in the metal-poor models, $[\mathrm{Fe} / \mathrm{H}] \lesssim-2 \mathrm{dex}$, it is the over-ionisation driven by a hard UV radiation field that acts on the line opacity and thereby counteracts the NLTE effects on the source function. We have masked out all Ti I lines (see Sect. 2.3), so differences in $[\mathrm{Ti} / \mathrm{Fe}]$ are small $\lesssim 0.06 \mathrm{dex}$ and represent indirect NLTE effects on other stellar parameters. The difference in $[\mathrm{Mn} / \mathrm{Fe}]$ is shown only for a few metal-rich clusters, and it is increasing to lower $[\mathrm{Fe} / \mathrm{H}]$.

Figures 4 and 5 show our NLTE and LTE results, respectively for the 12 clusters in the $T_{\text {eff }}-\log g$ plane. We also overlay the PARSEC (Marigo et al. 2017) and Victoria-Regina (VandenBerg et al. 2014; hereafter VR) isochrones to facilitate the analysis of the evolutionary stages probed by the stellar sample. The VR isochrones assume the He abundance of $Y=0.26$ and an $\alpha$ enhancement, as given by our measurements of $[\mathrm{Mg} / \mathrm{Fe}]$. The PARSEC isochrones are computed using an effective metallicity (Aldo Serenelly, priv. comm.)

$Z=Z_{0}\left(0.659 f_{\alpha}+0.341\right)$,

where $Z_{0}=10^{[\mathrm{Fe} / \mathrm{H}]}$ and $f_{\alpha}=10^{[\mathrm{Mg} / \mathrm{Fe}]}$. The error of the spectroscopic estimates is shown in the inset and it represents the typical uncertainty of our analysis $\left(\Delta\left(T_{\text {eff }}\right)=150 \mathrm{~K}\right.$ and $\Delta(\log (g))=$ 0.3 dex based on analysis of Gaia-ESO benchmark stars). The star-to-star scatter in the $T_{\text {eff }}-\log (g)$ plane is very small, and, within the uncertainties, consistent with the isochrones.

Surprisingly, both NLTE and LTE spectroscopic parameters agree well with the isochrones computed for the corresponding $[\mathrm{Fe} / \mathrm{H}]$, despite the large differences between NLTE and LTE parameters $\left(T_{\text {eff }}, \log g,[\mathrm{Fe} / \mathrm{H}]\right.$, and $\left.[\mathrm{Mg} / \mathrm{Fe}]\right)$ especially at low metallicity. This would appear counter-intuitive, at first glance, given the large offsets demonstrated in Fig. 3. However, this effect is, in fact, simply a result of the complex correlations in stellar parameters (as also extensively discussed in Ruchti et al. 2013): NLTE effects in the over-ionisation - dominated species (such as Fe I, Ti I) significantly change the excitation and ionisation balance, such that the theoretical spectral lines tend to be weaker and a higher abundance would be inferred by comparing them to the observed spectra. Consequently, larger estimates of $T_{\text {eff }}, \log g$, and $[\mathrm{Fe} / \mathrm{H}]$ are expected from the NLTE modelling compared to LTE (see also Lind et al. 2012). The difference between NLTE and LTE $[\mathrm{Fe} / \mathrm{H}]$ estimates is exactly the offset needed to match the higher (lower) $T_{\text {eff }}$ and higher (lower) $\log g$ to the corresponding isochrone computed for the NLTE (LTE) metallicity and $\alpha$-enhancement. This suggests that even large systematic errors in spectroscopic estimates may remain undetected in the $T_{\text {eff }}-\log g$ plane, when spectroscopic values are gauged by comparing them with isochrones.

In Fig. 6 we show examples of spectral fits for two stars randomly selected from our sample of clusters. Both LTE and NLTE model spectra match the observed ones very well, having similar $\chi_{r}^{2}$, while the fit residuals mostly show noise and data reduction artefacts.

Our LTE and NLTE results show a slight tendency towards a hotter $T_{\text {eff }}$ scale, which may appear more consistent with the PARSEC models. However, it might be premature to draw more specific conclusions on this matter, as we are aware of the imperfections of the stellar atmosphere and spectral model grids, such as an approximate treatment of convection as well as calibrations that are employed in the stellar evolution models (e.g. Fu et al. 2018). At this stage, it appears to be sufficient to emphasise that our spectroscopic results are internally consistent, and allow predictive statements to be made on the astrophysical significance of the similarities and/or differences of chemical abundance patterns in the clusters.

\subsubsection{Error estimates}

To explore the sensitivity of the abundances to the uncertainties in stellar parameters, we use a method similar to the one employed in Bergemann et al. (2017a). The standard errors are estimated by comparison with the independent stellar parameters for the benchmark stars (Sect. 3.1). These are $\pm \Delta T_{\text {eff }}=150 \mathrm{~K}$, $\pm \Delta \log (g)=0.3 \mathrm{dex}$ and $\pm \Delta[\mathrm{Fe} / \mathrm{H}]=0.1 \mathrm{dex}$. For $V_{\text {mic }}$, we use the uncertainty of $\pm 0.2 \mathrm{~km} \mathrm{~s}^{-1}$. We perturb one parameter at a time by its standard error, and re-determine the abundance of an element, while keeping the parameter fixed during the $\chi^{2}$ optimisation. We then compare the resulting abundance with the estimate obtained from the full solution, when all labels are solved for simultaneously. Table A.1 presents the resulting uncertainties for five stars representative of the sample. These differences are added in quadrature and are used as a measure of the systematic error of abundances $\Delta X$. The systematic errors derived using this procedure are typically within $0.10-0.15$ dex (Table A.1).

The test of internal accuracy suggests (Sect. 2.4) that we cannot have derived robust $\mathrm{Mn}$ abundances for much of the parameter space, because Mn lines in the HR10 spectra are weak 

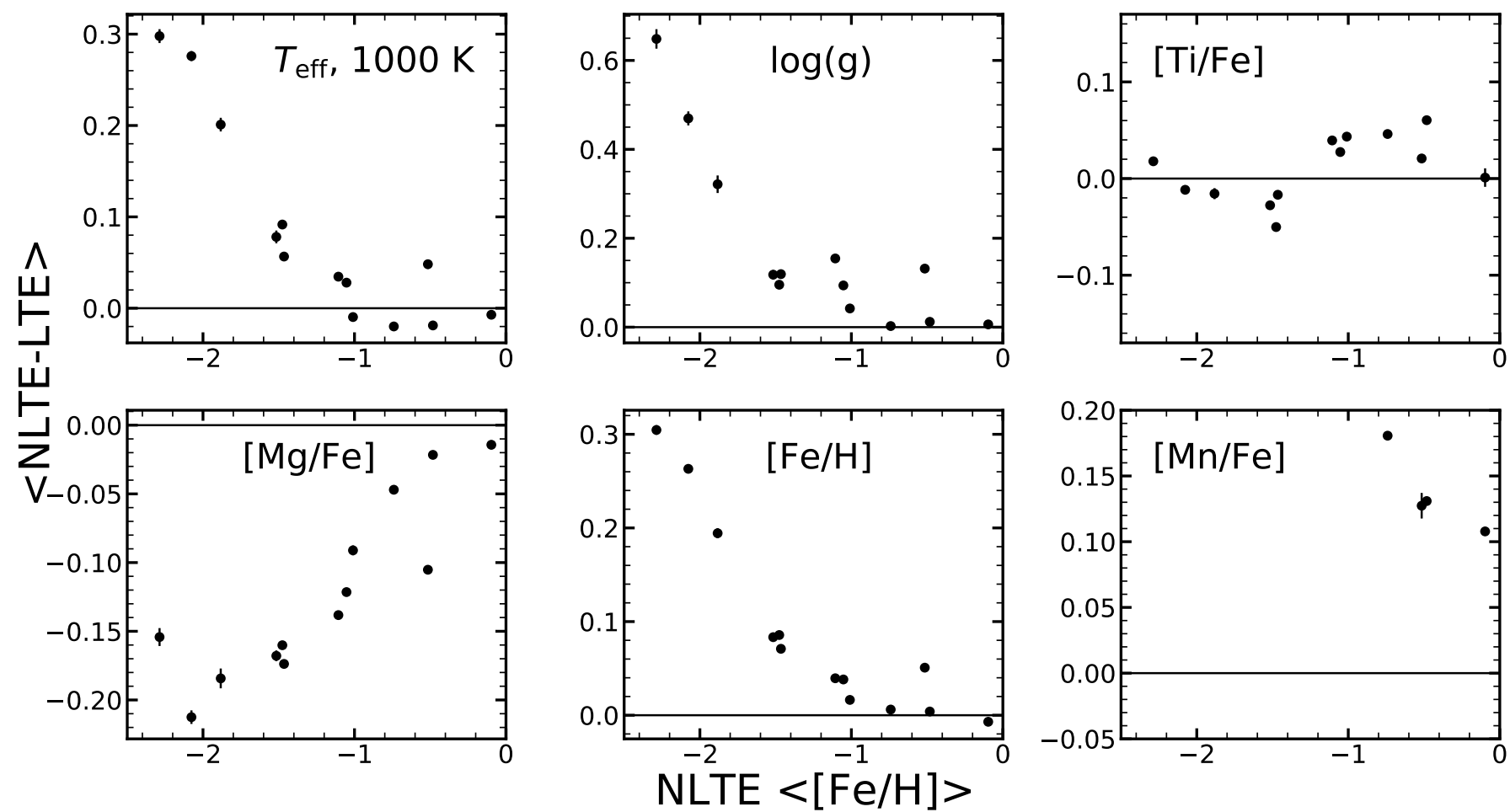

Fig. 3. Mean differences between NLTE and LTE parameters for stars within each cluster against NLTE metallicity. For [Mn/Fe] only clusters with $[\mathrm{Fe} / \mathrm{H}]>-1$ dex are shown. See Sect. 3.2.2 for details.

in the metal-poor regime. Hence, the mean $[\mathrm{Mn} / \mathrm{Fe}]$ ratios are only provided for the two metal-rich clusters NGC 3532 and NGC 5927.

\subsubsection{Abundance spreads in clusters}

Figure 7 shows the $[\mathrm{Fe} / \mathrm{H}],[\mathrm{Ti} / \mathrm{Fe}]$, and $[\mathrm{Mg} / \mathrm{Fe}]$ abundance estimates in stars of OCs and GCs against stellar $T_{\text {eff. The uncertain- }}$ ties represent the systematic errors computed as described in see Sect. 3.2.3. The open cluster NGC 2243 is shown separately in Fig. 8 as it shows signatures of atomic diffusion. Of a particular interest is the dip of $[\mathrm{Fe} / \mathrm{H}]$ at the cluster TO $\left(T_{\text {eff }} \sim 6400 \mathrm{~K}\right)$, which is qualitatively consistent with the predictions of stellar evolution models, which include radiative acceleration and gravitational settling (e.g. Deal et al. 2018). We leave a detailed exploration of this effect for our future study.

Whereas prominent systematic biases appear to be absent for most clusters, there is some evidence for a small anti-correlation of $[\mathrm{Mg} / \mathrm{Fe}]$ and/or $[\mathrm{Ti} / \mathrm{Fe}]$ values with $T_{\text {eff }}$, for the moderately metal-poor clusters NGC 1851, NGC 362, M 2, and NGC 6752. These clusters also show a somewhat tilted distribution of stars relative to the isochrones in the $T_{\text {eff }}-\log g$ plane (Figs. 4 and 5) suggesting that the origin of the trends is likely in the spectral models or method, employed in this work. Currently we have no straightforward solution for this effect.

The average abundance of a cluster $\langle X\rangle$ and internal dispersion $\sigma_{X}$ are computed using maximum likelihood (ML) approach (Walker et al. 2006; Piatti \& Koch 2018), where we take into account the individual abundance uncertainties $\Delta X$ of each star. We numerically maximise the logarithm of the likelihood $L$, given as:

$\ln L=-\frac{1}{2} \sum_{i}^{N} \ln \left(\Delta X_{i}^{2}+\sigma_{X}^{2}\right)-\frac{1}{2} \sum_{i}^{N} \frac{\left(X_{i}-\langle X\rangle\right)^{2}}{\Delta X_{i}^{2}+\sigma_{X}^{2}}-\frac{N}{2} \ln 2 \pi$, where $N$ is the number of stars in a cluster and $X$ refers to one of $[\mathrm{Fe} / \mathrm{H}],[\mathrm{Mg} / \mathrm{Fe}],[\mathrm{Ti} / \mathrm{Fe}]$ and $[\mathrm{Mn} / \mathrm{Fe}]$. The errors of the mean and dispersion are computed from the respective covariance matrices (Walker et al. 2006).

We find that all clusters are homogeneous in $[\mathrm{Fe} / \mathrm{H}]$ and $[\mathrm{Ti} / \mathrm{Fe}]$ at an uncertainty level of 0.03 dex. Four clusters (M 15, M 2, NGC 4833, NGC 2808) show a larger scatter in $[\mathrm{Mg} / \mathrm{Fe}]$ at the level of 0.07 dex or greater. Modest internal dispersions $\sigma_{[\mathrm{Mg} / \mathrm{Fe}]} \sim 0.04$ dex are detected in NGC 1904 and NGC 6572 .

Spreads in light element abundances, including $\mathrm{Mg}$, have already been reported for a number of clusters, including NGC 2808 (Carretta 2015), M 2 (Yong et al. 2015), NGC 4833 (Carretta et al. 2014) and M15 (Carretta et al. 2009). These spreads are typically attributed to multiple episodes of star formation and self-enrichment (see the recent review by Bastian \& Lardo 2018, and references therein).

The estimated internal dispersions are summarised in Table A.4. In the following, to be consistent with the literature, we will focus on the observed intra-cluster dispersion, instead of the ML estimated internal dispersion. We note that these two are not the same as the latter probes the intrinsic dispersion that is not accounted for by the measurement uncertainties, while the former includes both.

\section{Discussion}

\subsection{Comparison with the literature}

In what follows, we discuss our results for the Galactic clusters in the context of their chemical properties. Many literature abundances are given in "standard" format: mean \pm intra-cluster spread, computed as a simple standard deviation using all measurement in the cluster. In some cases, when not given in the same format, we recompute the mean and the standard deviations using the values of individual stars in the literature. Our 

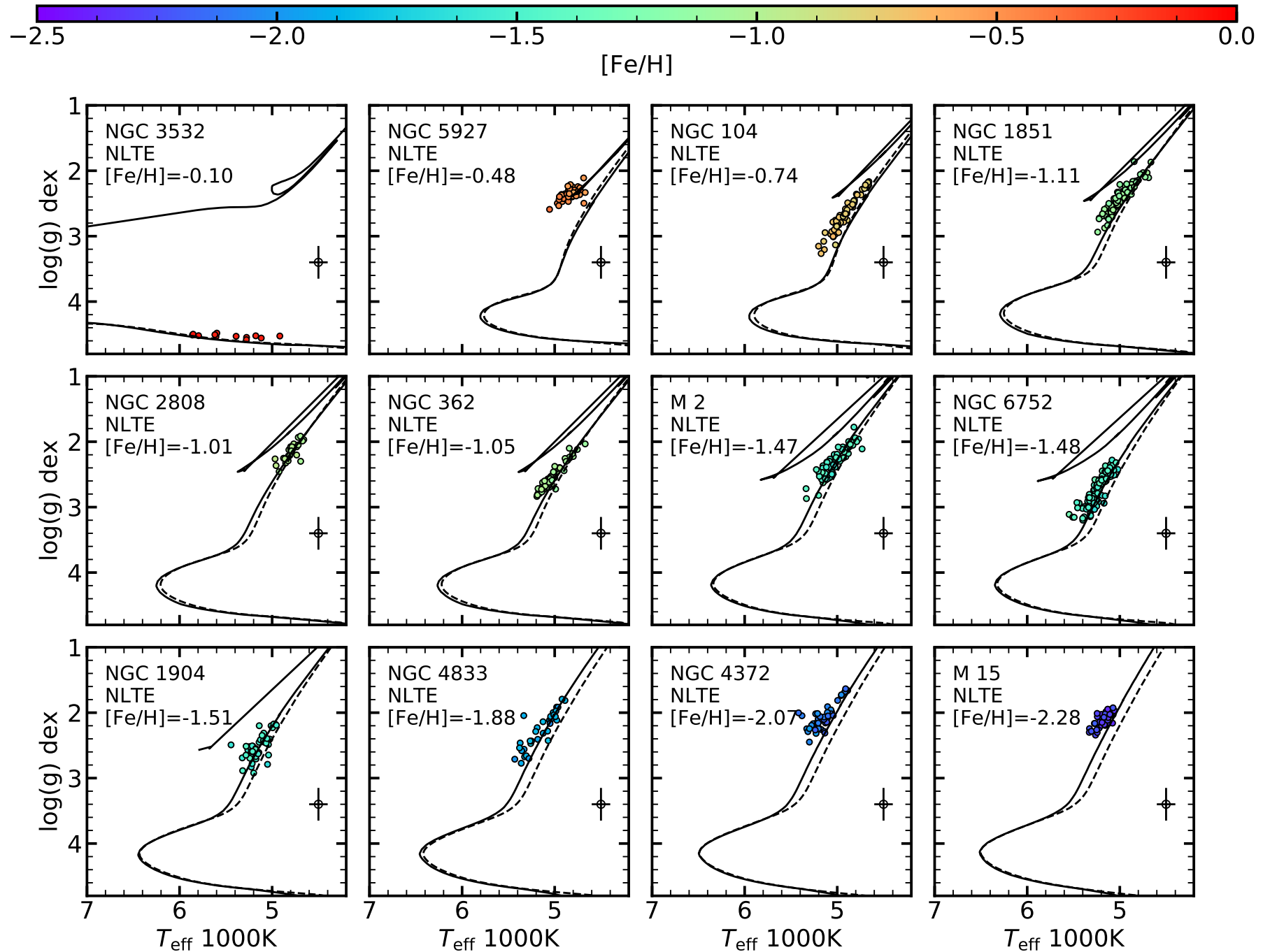

Fig. 4. NLTE spectroscopic parameters compared with the PARSEC (solid line) and Victoria-Regina (dashed line) isochrones (Marigo et al. 2017; VandenBerg et al. 2014). The isochrones were computed using literature ages and $[\mathrm{Fe} / \mathrm{H}]$ from our NLTE analysis. The colour of the points indicates their $[\mathrm{Fe} / \mathrm{H}]$. We note the different target selection for NGC 5927, where the observed stellar sample contains mostly red clump stars.

own results are presented in the same format with mean from ML analysis and the observed intracluster spread (not the ML estimated internal dispersion) given in Table 2.

We start with two open clusters and then continue with globular clusters, in order from the most metal-rich to the most metalpoor one.

\subsubsection{NGC 3532}

NGC 3532 is a young nearby metal-rich cluster at a heliocentric distance of $d_{\odot} \sim 0.5 \mathrm{kpc}$ (Clem et al. 2011; Fritzewski et al. 2019). The cluster has been extensively surveyed for white dwarfs (Dobbie et al. 2009, 2012) which has allowed accurate estimate of the cluster age of $\sim 300 \mathrm{Myr}$ from the white dwarf cooling sequence.

On the basis of 12 main-sequence stars, we find the metallicity $[\mathrm{Fe} / \mathrm{H}]_{\mathrm{NLTE}}=-0.10 \pm 0.02 \mathrm{dex}$ and $[\mathrm{Fe} / \mathrm{H}]_{\mathrm{LTE}}=-0.09 \pm$ 0.03 dex. This estimate is consistent, within the uncertainties, with estimates based on the analysis of high-resolution spectra by Santos et al. (2012), Conrad et al. (2014), and Netopil (2017). Fritzewski et al. (2019) reported the metallicity of $[\mathrm{Fe} / \mathrm{H}]$ of $-0.07 \pm 0.10$ dex using lower-resolution near-IR spectra.
Our NLTE abundance ratios suggest that the cluster is moderately $\alpha$-poor, with $[\mathrm{Mg} / \mathrm{Fe}]_{\mathrm{NLTE}}$ of $-0.09 \pm 0.01 \mathrm{dex}$, although the $[\mathrm{Ti} / \mathrm{Fe}]$ ratio is solar $[\mathrm{Ti} / \mathrm{Fe}]_{\text {NLTE }}=0.01 \pm 0.03 \mathrm{dex}$. The $[\mathrm{Mn} / \mathrm{Fe}]$ ratio is sub-solar, $[\mathrm{Mn} / \mathrm{Fe}]_{\mathrm{NLTE}}=-0.16 \pm 0.03 \mathrm{dex}$.

\subsubsection{NGC 2243}

NGC 2243 is an old Galactic open cluster located below the Galactic plane, at $z=-1.1 \mathrm{kpc}$, and at a Galactocentric distance of $10.7 \mathrm{kpc}$ (Jacobson et al. 2011). The age of the cluster was determined by several methods including spectroscopy, CMD isochrone fitting (Anthony-Twarog et al. 2005), using model age-luminosity and age-radius relations for eclipsing binaries (Kaluzny et al. 2006), bracketing $4 \pm 1$ Gyr.

The cluster has been subject to a very detailed chemical abundance analysis (for example the review by Heiter et al. 2014). Gratton (1982) and Gratton \& Contarini (1994) derived a spectroscopic metallicity of $[\mathrm{Fe} / \mathrm{H}]=-0.42 \pm 0.05$, as well as detailed chemical abundances of the elements from $\mathrm{C}$ to $\mathrm{Eu}$ for a few RGB stars in the cluster. Their estimates were confirmed by Friel et al. (2002) and Jacobson et al. (2011), who derived $\mathrm{Fe}, \mathrm{Ni}, \mathrm{Ca}, \mathrm{Si}, \mathrm{Ti}, \mathrm{Cr}, \mathrm{Al}, \mathrm{Na}$, and $\mathrm{Mg}$ abundances in a small 


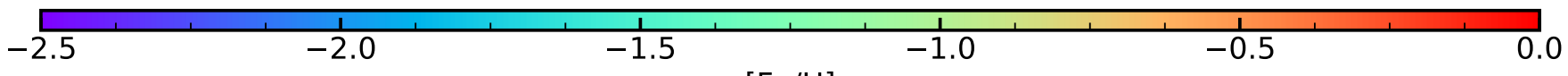

$[\mathrm{Fe} / \mathrm{H}]$
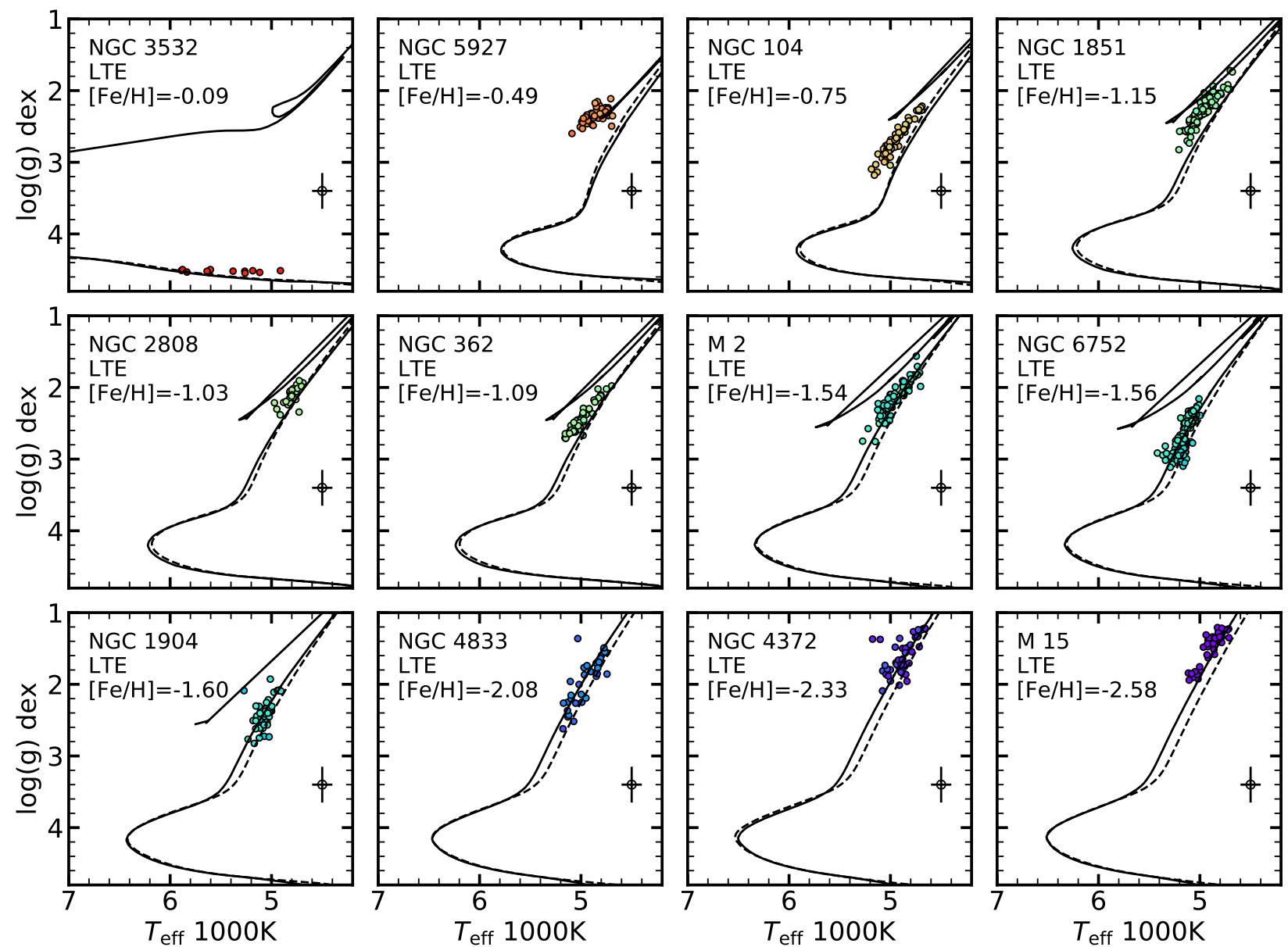

Fig. 5. LTE spectroscopic parameters compared with the PARSEC (solid line) and Victoria-Regina (dashed line) isochrones (Marigo et al. 2017; VandenBerg et al. 2014). The isochrones were computed using literature ages and $[\mathrm{Fe} / \mathrm{H}]$ from our LTE analysis. The colour of the points indicates their $[\mathrm{Fe} / \mathrm{H}]$. We note the different target selection for NGC 5927, where the observed stellar sample contains mostly red clump stars.

sample of RGB stars. According to the latter study, this is one of the most metal-poor clusters at its location at an $R_{\mathrm{GC}} \sim$ $11 \mathrm{kpc}$. This cluster has also been observed within the OCCAM APOGEE survey (Cunha et al. 2016). Their estimates of abundances in NGC 2243 are somewhat different from Jacobson et al. (2011), with Mg being -0.14 dex lower and more subtle differences for the other elements. In contrast to Jacobson et al. (2011), Cunha et al. (2016) also find a very large spread of metallicities in the cluster members, ranging from -0.4 to +0.3 dex.

François et al. (2013) reported detailed abundances for the main-sequence and subgiant stars in the cluster. Their $[\mathrm{Fe} / \mathrm{H}]$ of $-0.54 \pm 0.10 \mathrm{dex}$ is consistent with our NLTE estimate of $[\mathrm{Fe} / \mathrm{H}]_{\mathrm{NLTE}}=-0.52 \pm 0.06 \mathrm{dex}$. Our estimate of $[\mathrm{Ti} / \mathrm{Fe}]_{\mathrm{NLTE}}=$ $0.02 \pm 0.08 \mathrm{dex}$ is also in agreement with the value obtained by François et al. (2013), $[\mathrm{Ti} / \mathrm{Fe}]=0.20 \pm 0.22 \mathrm{dex}$, within the combined uncertainties of both measurements. In fact, our lower estimate of $[\mathrm{Ti} / \mathrm{Fe}]$ corroborates the scaled-solar estimates of other $\alpha$-elements reported by François et al. (2013), $[\mathrm{Ca} / \mathrm{Fe}]=0.00 \pm 0.14 \mathrm{dex}$ and $[\mathrm{Si} / \mathrm{Fe}]=0.12 \pm 0.20 \mathrm{dex}$.

\subsubsection{NGC 5927}

NGC 5927 is a metal-rich globular cluster located close to the Galactic plane, at an altitude $z \sim 0.6 \mathrm{kpc}$ (Casetti-Dinescu et al.
2007). With an age of 12 Gyr (Dotter et al. 2010) and metallicity of $[\mathrm{Fe} / \mathrm{H}] \sim-0.5$ dex (Mura-Guzmán et al. 2018), the cluster is among the oldest metal-rich clusters known in the Galaxy. High-resolution spectroscopy of the cluster revealed the presence of multiple populations, especially prominent in the anti-correlation between $\mathrm{Na}$ and $\mathrm{O}$ (Pancino et al. 2017a; Mura-Guzmán et al. 2018). The latter study also pointed out a similarity in the chemical properties of NGC 5927 and NGC 6440, a metal-rich GC in the Galactic bulge that could potentially hint at the common origin of the both systems.

Our NLTE estimate $[\mathrm{Fe} / \mathrm{H}]_{\mathrm{NLTE}}=-0.48 \pm 0.05 \mathrm{dex}$ is in very good agreement with earlier spectroscopic studies (Mura-Guzmán et al. 2018, $[\mathrm{Fe} / \mathrm{H}]=-0.47 \pm 0.02$ dex). However, the abundance ratios are somewhat different. In particular, we find both $\mathrm{Mg}$ and $\mathrm{Ti}$ to be higher, $[\mathrm{Mg} / \mathrm{Fe}]_{\mathrm{NLTE}}=$ $0.39 \pm 0.04 \mathrm{dex}$ and $[\mathrm{Ti} / \mathrm{Fe}]_{\mathrm{NLTE}}=0.29 \pm 0.06 \mathrm{dex}$, compared to the results of the latter study. For Ti, our higher estimate is likely the consequence of NLTE over-ionisation, as the LTE abundance is $[\mathrm{Ti} / \mathrm{Fe}]_{\mathrm{LTE}}=0.23 \pm 0.05 \mathrm{dex}$, which is consistent with the estimate of $[\mathrm{Ti} / \mathrm{Fe}]=0.32 \pm 0.05$ dex from Mura-Guzmán et al. (2018). In contrast, the difference in $\mathrm{Mg}$ abundance is not related to NLTE. Our LTE Mg abundance is $[\mathrm{Mg} / \mathrm{Fe}]_{\text {LTE }}=0.41 \pm 0.05 \mathrm{dex}$, which is much higher than that of Mura-Guzmán et al. (2018), $[\mathrm{Mg} / \mathrm{Fe}]=0.27 \pm 0.02 \mathrm{dex}$. It is 


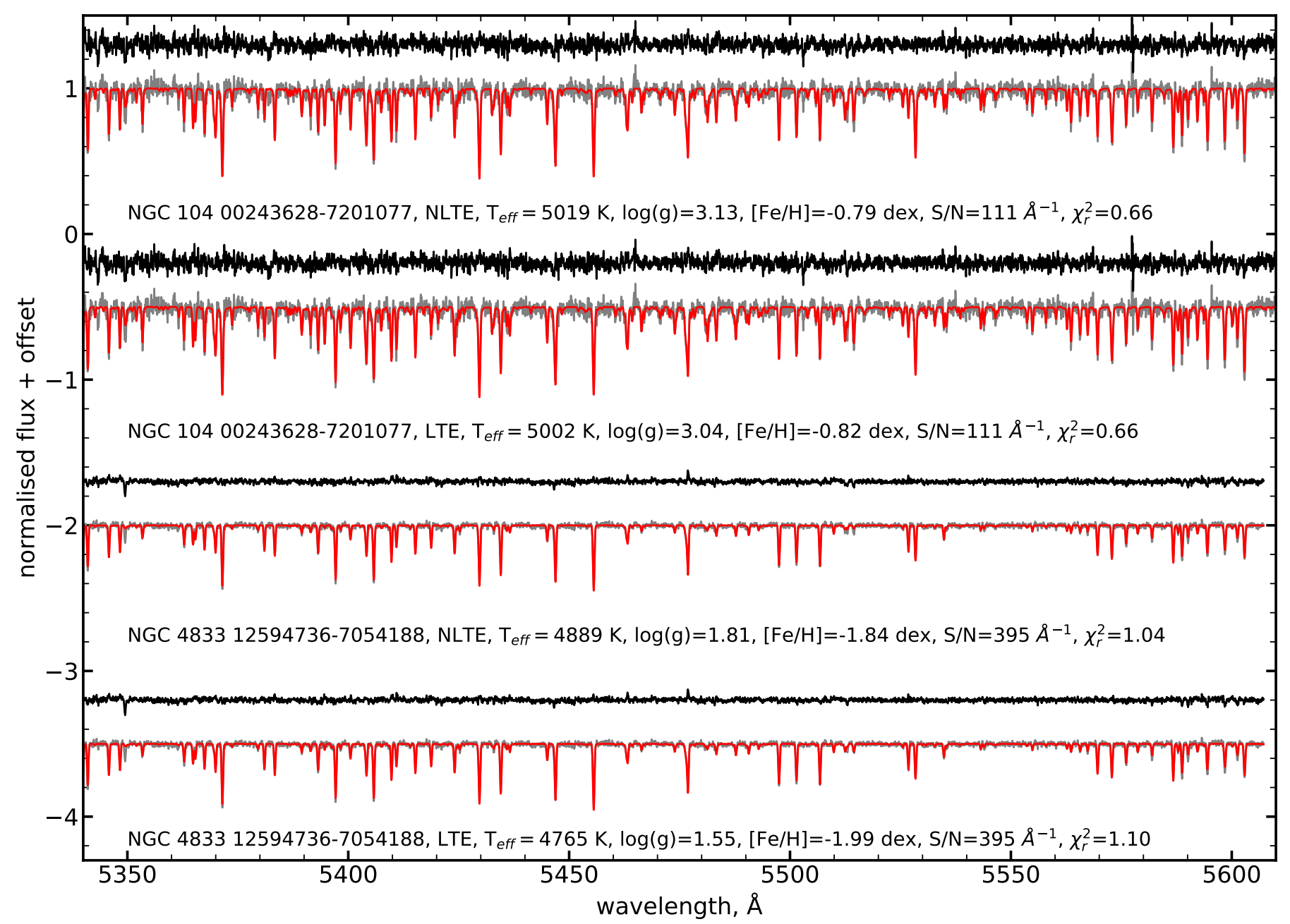

Fig. 6. NLTE and LTE spectral fits for two stars from our cluster sample. The best-fit spectra are shown as red lines. The observed spectra are depicted with grey lines, while the fit residuals are shown with black lines. See inset for the derived $T_{\text {eff }}, \log (g)$, $[\mathrm{Fe} / \mathrm{H}]$, reduced $\chi^{2}$, and the $\mathrm{S} / \mathrm{N}$ of the observed spectrum.

possible that the differences stem from the differences in atomic data and/or model atmospheres. Mura-Guzmán et al. (2018) employ the MOOG code, Kurucz model atmospheres, and linelists from Villanova \& Geisler (2011) and references therein. Our linelists have been extensively updated over the past years, and in particular for $\mathrm{Mg}$ lines, we used the data from Pehlivan Rhodin et al. (2017). We were unable to find the atomic data in Villanova \& Geisler (2011) and hence cannot provide a detailed analysis of the consistency of the models. Our average $[\mathrm{Mn} / \mathrm{Fe}]$ abundance ratio in NGC 5927 is subsolar $[\mathrm{Mn} / \mathrm{Fe}]_{\mathrm{NLTE}}=-0.20 \pm 0.03 \mathrm{dex},[\mathrm{Mn} / \mathrm{Fe}]_{\mathrm{LTE}}=-0.34 \pm$ 0.03 dex. This estimate is much lower compared to $[\mathrm{Mn} / \mathrm{Fe}]=$ $-0.09 \pm 0.08$ dex derived by Mura-Guzmán et al. (2018), but it is mostly due to the difference of -0.16 in the adopted solar abundance $\left(\log A(\mathrm{Mn})_{\odot}=5.37 \mathrm{dex}\right.$ and $\log A(\mathrm{Mn})_{\odot}=5.53 \mathrm{dex}$, respectively).

\subsubsection{NGC 104 (47 Tuc)}

NGC 104 (47 Tuc) is among the brightest and most well-studied clusters in the Milky Way (e.g. Anderson et al. 2009; Campos et al. 2018; Milone et al. 2012; Lapenna et al. 2014; Cordero et al. 2014; Thygesen et al. 2014; Černiauskas et al. 2017). The recent estimate of the distance to the cluster is $d_{\odot}=4.45 \mathrm{kpc}$
(Chen et al. 2018), which was obtained on the basis of Gaia DR2 parallaxes. The reddening towards the system is very low $E(B-$ $V)=0.03 \pm 0.10 \mathrm{mag}$ allowing an accurate estimate of the cluster age of $12.5 \mathrm{Gyr}$ (Brogaard et al. 2017). Chemical abundance patterns, in the form of $\mathrm{Na}-\mathrm{O}$ anti-correlations, enrichment in $\mathrm{He}$ and $\mathrm{N}$, and depletion of $\mathrm{C}$, indicate complex chemical evolution in the cluster (Cordero et al. 2014; Kučinskas et al. 2014; Marino et al. 2016).

Our NLTE estimate of the cluster metallicity, $[\mathrm{Fe} / \mathrm{H}]_{\mathrm{NLTE}}=$ $-0.74 \pm 0.03 \mathrm{dex}$, is in very good agreement with previous estimates (Koch \& McWilliam 2008; Cordero et al. 2014; Dobrovolskas et al. 2014; Thygesen et al. 2014). The latter study reports $[\mathrm{Fe} / \mathrm{H}]=-0.78 \pm 0.07$ dex obtained by $1 \mathrm{D}$ LTE modelling of $\mathrm{Fe}$ lines. The authors also test the effect of NLTE, finding the effects to be of the order +0.02 dex on the Fe abundances. Indeed, this is fully confirmed by our LTE metallicities, which are 0.01 dex lower compared to our NLTE results. For $\mathrm{Mg}$, Thygesen et al. (2014) report $[\mathrm{Mg} / \mathrm{Fe}]=0.44 \pm 0.05 \mathrm{dex}$ in LTE, which is in excellent agreement with our LTE value, $[\mathrm{Mg} / \mathrm{Fe}]_{\text {LTE }}=0.42 \pm 0.04 \mathrm{dex}$, and is only slightly higher than our NLTE result $[\mathrm{Mg} / \mathrm{Fe}]_{\mathrm{NLTE}}=0.38 \pm 0.05 \mathrm{dex}$. Also the Ti abundances are consistent with Thygesen et al. (2014). We obtain $[\mathrm{Ti} / \mathrm{Fe}]_{\mathrm{NLTE}}=0.30 \pm 0.07 \mathrm{dex}$ and $[\mathrm{Ti} / \mathrm{Fe}]_{\mathrm{LTE}}=$ $0.26 \pm 0.07 \mathrm{dex}$, which agrees within the uncertainties with the 

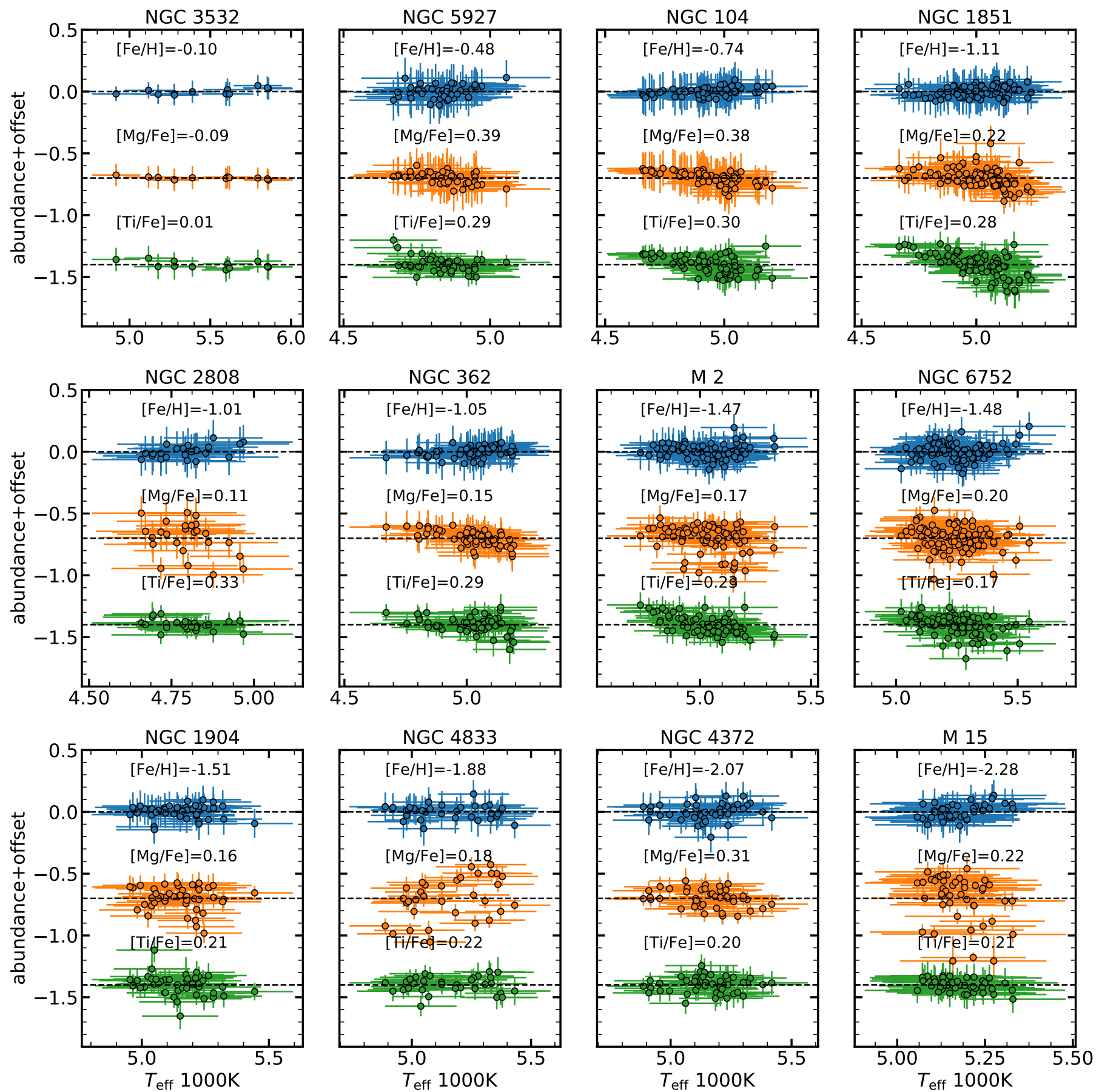

Fig. 7. NLTE abundances as a function of $T_{\text {eff }}$ for all cluster stars. The average is shown for all elements. The scatter in $[\mathrm{Mg} / \mathrm{Fe}]$ is much larger than in $[\mathrm{Fe} / \mathrm{H}]$ and $[\mathrm{Ti} / \mathrm{Fe}]$, and it is typically attributed to multiple episodes of star formation and self-enrichment (see the recent review by Bastian \& Lardo 2018, and references therein). See Sect. 3.2.4 for details.

measured value of $[\mathrm{Ti} / \mathrm{Fe}]=0.28 \pm 0.08$ dex from Thygesen et al. (2014).

\subsubsection{NGC 1851}

NGC 1851 is a moderately metal-poor globular cluster located at an $R_{\mathrm{GC}}$ of $17 \mathrm{kpc}$ from the Galactic centre and $\sim 7 \mathrm{kpc}$ below the disk plane (Harris 1996, 2010 edition). Wagner-Kaiser et al. (2017) find a cluster age of 11.5 Gyr. Some have argued for an evolutionary connection between NGC 1851 and several other clusters (NGC 1904, NGC 2808, and NGC 2298) on the basis of their spatial proximity (Bellazzini et al. 2001), as we confirm by our abundances below. An idea has been put forward that all four clusters are associated with the disrupted Canis Major dwarf galaxy (Martin et al. 2004). Others suggest that NGC 1851 is possibly the nucleus of a disrupted dwarf galaxy (Bekki \& Yong 2012; Kuzma et al. 2018) or could have formed as a result of the merger of two globular clusters (Carretta et al. 2011). The cluster hosts multiple stellar populations, seen in photometric data on the main sequence, subgiant branch, and RGB (Milone et al. 2008; Turri et al. 2015; Cummings et al. 2017). Also the spectroscopic analysis of $\mathrm{C}$ and $\mathrm{N}$ suggests the presence of several 

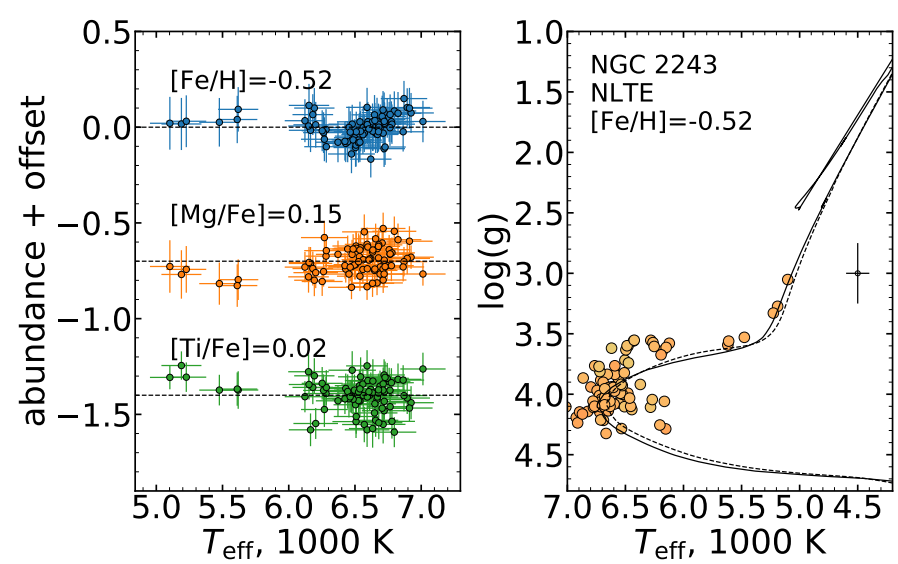

Fig. 8. Abundances as a function of $T_{\text {eff }}$ and the $T_{\text {eff }}-\log (g)$ diagram for the open cluster NGC 2243. All values are our NLTE results. The isochrones were computed for the age of $3.8 \mathrm{Gyr}$ from Anthony-Twarog et al. (2005) and $[\mathrm{Fe} / \mathrm{H}]_{\mathrm{NLTE}}=-0.52$ dex.

populations (Yong \& Grundahl 2008; Yong et al. 2015; Simpson et al. 2017).

Our metallicities for NGC 1851 are slightly higher compared to previous studies. Gratton et al. (2012) find a range of metallicities in the cluster from $[\mathrm{Fe} / \mathrm{H}]=-1.23 \pm 0.06 \mathrm{dex}$ (subgiant branch) to $[\mathrm{Fe} / \mathrm{H}]=-1.14 \pm 0.06 \mathrm{dex}(\mathrm{RGB})$. Our analysis yields $[\mathrm{Fe} / \mathrm{H}]_{\mathrm{NLTE}}=-1.11 \pm 0.04 \mathrm{dex}$ and $[\mathrm{Fe} / \mathrm{H}]_{\mathrm{LTE}}=-1.15 \pm$ 0.04 dex, whereas Yong et al. $(2015)$ report $[\mathrm{Fe} / \mathrm{H}]=-1.28 \pm$ 0.05 and Marino et al. $(2014)$ obtain $[\mathrm{Fe} / \mathrm{H}]=-1.33 \pm 0.09$ dex.

For $\mathrm{Mg}$, we find $[\mathrm{Mg} / \mathrm{Fe}]_{\mathrm{NLTE}}=0.22 \pm 0.08 \mathrm{dex}$, which is lower than the value reported by Marino et al. (2014) $[\mathrm{Mg} / \mathrm{Fe}]=$ $0.44 \pm 0.16$ dex. However, this difference can be almost entirely explained by NLTE. Indeed our LTE estimates of $[\mathrm{Mg} / \mathrm{Fe}]$ are much higher, $[\mathrm{Mg} / \mathrm{Fe}]_{\mathrm{LTE}}=0.36 \pm 0.05 \mathrm{dex}$, and are also in agreement with the LTE estimates by Carretta et al. (2011), $[\mathrm{Mg} / \mathrm{Fe}]=0.35 \pm 0.03 \mathrm{dex}$. For Ti, we find the opposite offset, in the sense that our NLTE values, $[\mathrm{Ti} / \mathrm{Fe}]_{\mathrm{NLTE}}=0.28 \pm 0.06 \mathrm{dex}$, are higher compared to the LTE results by Carretta et al. (2011) $[\mathrm{Ti} / \mathrm{Fe}]=0.17 \pm 0.05 \mathrm{dex}$. This can be explained by NLTE, as our LTE abundances of Ti are slightly lower, $[\mathrm{Ti} / \mathrm{Fe}]_{\mathrm{LTE}}=$ $0.24 \pm 0.06 \mathrm{dex}$, consistent with the latter study within the combined uncertainties of the both LTE measurements.

It is interesting, in the context of the common formation scenario of NGC 1851 and NGC 2808, as proposed by Martin et al. (2004), that our chemical abundances in the two clusters are very similar. In fact, given the uncertainties of our measurements, both clusters are consistent with being formed from the same material, and having the same progenitor system.

\subsubsection{NGC 2808}

NGC 2808, a moderately metal-poor old cluster, is among the most massive and complex systems in the Milky Way galaxy (Simioni et al. 2016), with the mass of $7.42 \times 10^{5} M_{\odot}$ (Baumgardt \& Hilker 2018) and multiple populations (Piotto et al. 2007; Milone et al. 2015). NGC 2808 was among the first clusters, for which a prominent $\mathrm{Na}-\mathrm{O}$ anti-correlation was reported (Carretta et al. 2006), along with a He spread (D'Antona et al. 2005) and a $\mathrm{Mg}-\mathrm{Al}$ anti-correlation (Carretta 2006).

Our LTE metallicity, $[\mathrm{Fe} / \mathrm{H}]_{\mathrm{LTE}}=-1.03 \pm 0.05 \mathrm{dex}$, is slightly higher compared to the recent literature values. Carretta (2015) report $[\mathrm{Fe} / \mathrm{H}]=-1.13 \pm 0.03$ dex using the Fe I lines and $[\mathrm{Fe} / \mathrm{H}]=-1.14 \pm 0.03$ dex using the Fe II lines. They also find a large spread in $[\mathrm{Mg} / \mathrm{Fe}]$ abundance ratios, which is corroborated by our results. In particular, we find that the individual LTE abundance ratios of $[\mathrm{Mg} / \mathrm{Fe}]$ range from 0.08 to $0.45 \mathrm{dex}$, and the average value and its dispersion, $[\mathrm{Mg} / \mathrm{Fe}]_{\mathrm{LTE}}=0.22 \pm 0.15 \mathrm{dex}$, is consistent with $[\mathrm{Mg} / \mathrm{Fe}]=0.26 \pm 0.16$ dex obtained by Carretta (2015). For Ti, our estimate $[\mathrm{Ti} / \mathrm{Fe}]_{\text {LTE }}=0.29 \pm 0.04 \mathrm{dex}$ is slightly higher compared to $[\mathrm{Ti} / \mathrm{Fe}]=0.21 \pm 0.04 \mathrm{dex}$ derived by Carretta (2015). Our NLTE measurements are: $[\mathrm{Fe} / \mathrm{H}]_{\mathrm{NLTE}}=-1.01 \pm 0.05 \mathrm{dex},[\mathrm{Mg} / \mathrm{Fe}]_{\mathrm{NLTE}}=0.11 \pm 0.14 \mathrm{dex}$, and $[\mathrm{Ti} / \mathrm{Fe}]_{\mathrm{NLTE}}=0.33 \pm 0.04 \mathrm{dex}$.

\subsubsection{NGC 362}

The globular cluster NGC 362 has been extensively studied in the literature since the early work by Menzies (1967). A recent analysis of Gaia DR2 astrometric data by Chen et al. (2018) places it at a heliocentric distance of $8.54 \mathrm{kpc}$, relatively close to the Galactic disk plane. Photometric studies of the cluster revealed multiple sequences on the HB (Dotter et al. 2010; Gratton et al. 2010; Piotto et al. 2012). Spectroscopic followup confirmed its unique nature, with discrete groups of $\mathrm{Na} / \mathrm{O}$ ratios (Carretta et al. 2013), a bimodal distribution of CN (Smith \& Langland-Shula 2009; Lim et al. 2016), a very large spread of Al abundances.

Our NLTE metallicity for this cluster, $[\mathrm{Fe} / \mathrm{H}]_{\mathrm{NLTE}}=-1.05 \pm$ $0.04 \mathrm{dex}$, is somewhat higher compared to the results of the earlier studies. Our LTE estimate is lower, $[\mathrm{Fe} / \mathrm{H}]_{\mathrm{LTE}}=-1.09 \pm$ 0.04 dex and is consistent with the RR Lyr-based value from Székely et al. (2007). A very careful analysis of high-resolution spectra by Worley \& Cottrell (2010) yielded $[\mathrm{Fe} / \mathrm{H}]=-1.20 \pm$ 0.09 (from the Fe II lines), which is consistent within the uncertainty with our LTE estimate. A somewhat lower value is reported by D'Orazi et al. (2015). They find $[\mathrm{Fe} / \mathrm{H}]$ of $-1.26 \mathrm{dex}$ from the LTE analysis of RGB stars. The perhaps most extensive chemical study of the cluster, to date, is that by Carretta et al. (2013) employing UVES and Giraffe spectra of 138 RGB stars. For the UVES sample, they find a mean LTE metallicity of $[\mathrm{Fe} / \mathrm{H}]=-1.17 \pm 0.05$ dex from the Fe I lines and $[\mathrm{Fe} / \mathrm{H}]=$ $-1.21 \pm 0.08$ dex from Fe II lines that is in agreement with our LTE metallicity. Their abundance of [Ti/Fe] $(0.22 \pm 0.04 \mathrm{dex}$ based on the UVES spectra) and $[\mathrm{Mg} / \mathrm{Fe}](0.33 \pm 0.04 \mathrm{dex})$ are also in good agreement with our LTE estimates, $[\mathrm{Ti} / \mathrm{Fe}]_{\mathrm{LTE}}=$ $0.26 \pm 0.06 \mathrm{dex}$ and $[\mathrm{Mg} / \mathrm{Fe}]_{\mathrm{LTE}}=0.26 \pm 0.06 \mathrm{dex}$. In contrast, our NLTE values are considerably different, $[\mathrm{Ti} / \mathrm{Fe}]_{\mathrm{NLTE}}=$ $0.29 \pm 0.06 \mathrm{dex}$ and $[\mathrm{Mg} / \mathrm{Fe}]_{\mathrm{NLTE}}=0.15 \pm 0.06 \mathrm{dex}$. To the best of our knowledge, this paper is the first study to provide estimates of NLTE abundances in this cluster.

\subsubsection{2 (NGC 7089)}

M 2 is an old cluster in the Galactic halo at a distance of $\sim 7 \mathrm{kpc}$ below the Galactic plane and at a heliocentric distance of $11.5 \mathrm{kpc}$ (Harris 1996, 2010 edition). The cluster was the first system, in which a $\mathrm{CN}$ distribution bimodality was detected (Smith \& Mateo 1990; Lardo et al. 2012, 2013). Yong et al. (2014) argued for a trimodal metallicity distribution that has been, however, disputed by Lardo et al. (2016), who found a bimodal distribution using Fe II lines. Milone et al. (2015) employed HST photometry to detect a very rich stellar environment, composed of three main populations distinguished by their metallicity and a spread in He abundance from the primordial mass fraction of $Y \sim 0.25$ to $Y \sim 0.31$. They also suggest that there are six sub-populations with unique light element abundance patterns, that could potentially hint at either an 
Table 2. Mean clusters abundances (ML estimate) with observed intra-cluster spread (standard deviation) and mean systematic error (err).

\begin{tabular}{lccccccc}
\hline \hline Cluster & $\begin{array}{c}\text { \#stars } \\
N\end{array}$ & $\begin{array}{c}{[\mathrm{Fe} / \mathrm{H}]_{\text {NLTE }} \text { dex }} \\
\text { avg std }\langle\text { err }\rangle\end{array}$ & $\begin{array}{c}{[\mathrm{Fe} / \mathrm{H}]_{\text {LTE }} \text { dex }} \\
\text { avg std }\langle\text { err }\rangle\end{array}$ & $\begin{array}{c}{[\mathrm{Mg} / \mathrm{Fe}]_{\text {NLTE }} \text { dex }} \\
\text { avg std }\langle\text { err }\rangle\end{array}$ & $\begin{array}{c}{[\mathrm{Mg} / \mathrm{Fe}]_{\text {LTE }} \text { dex }} \\
\text { avg std }\langle\text { err }\rangle\end{array}$ & $\begin{array}{c}{[\mathrm{Ti} / \mathrm{Fe}]_{\text {NLTE }} \text { dex }} \\
\text { avg std }\langle\text { err }\rangle\end{array}$ & $\begin{array}{c}{[\mathrm{Ti} / \mathrm{Fe}]_{\text {LTE dex }}} \\
\text { avg std }\langle\text { err }\end{array}$ \\
\hline NGC 3532 & 12 & -0.100 .020 .10 & -0.090 .030 .11 & -0.090 .010 .09 & -0.070 .010 .12 & 0.010 .030 .10 & 0.010 .030 .11 \\
NGC 5927 & 47 & -0.480 .050 .16 & -0.490 .050 .16 & 0.390 .040 .07 & 0.410 .050 .07 & 0.290 .060 .07 & 0.230 .050 .07 \\
NGC 2243 & 84 & -0.520 .060 .08 & -0.570 .070 .11 & 0.150 .070 .08 & 0.260 .090 .09 & 0.020 .080 .09 & 0.010 .090 .10 \\
NGC 104 & 68 & -0.740 .030 .15 & -0.750 .030 .17 & 0.380 .050 .08 & 0.420 .040 .08 & 0.300 .070 .08 & 0.260 .070 .08 \\
NGC 1851 & 88 & -1.110 .040 .14 & -1.150 .040 .15 & 0.220 .080 .10 & 0.360 .080 .08 & 0.280 .090 .11 & 0.240 .070 .10 \\
NGC 2808 & 25 & -1.010 .050 .14 & -1.030 .050 .15 & 0.110 .140 .08 & 0.220 .150 .06 & 0.330 .040 .07 & 0.300 .040 .07 \\
NGC 362 & 62 & -1.050 .040 .13 & -1.090 .040 .16 & 0.150 .060 .09 & 0.260 .070 .08 & 0.290 .060 .09 & 0.260 .060 .09 \\
M 2 & 78 & -1.470 .060 .09 & -1.540 .060 .12 & 0.170 .110 .09 & 0.340 .130 .08 & 0.230 .070 .10 & 0.250 .060 .09 \\
NGC 6752 & 110 & -1.480 .060 .09 & -1.560 .070 .12 & 0.200 .090 .09 & 0.350 .110 .09 & 0.170 .070 .09 & 0.230 .070 .10 \\
NGC 1904 & 44 & -1.510 .050 .09 & -1.600 .070 .12 & 0.160 .090 .09 & 0.310 .110 .09 & 0.210 .080 .10 & 0.240 .090 .10 \\
NGC 4833 & 33 & -1.880 .060 .08 & -2.080 .080 .11 & 0.180 .170 .08 & 0.360 .200 .10 & 0.220 .060 .08 & 0.240 .070 .10 \\
NGC 4372 & 45 & -2.070 .060 .09 & -2.340 .080 .13 & 0.310 .070 .09 & 0.510 .090 .09 & 0.200 .060 .08 & 0.220 .070 .10 \\
M 15 & 46 & -2.280 .060 .08 & -2.580 .070 .10 & 0.220 .190 .11 & 0.360 .230 .09 & 0.210 .050 .09 & 0.190 .050 .12 \\
\hline
\end{tabular}

independent enrichment and star formation history of the individual components or at a unique merger formation history of the cluster. The imaging data by Kuzma et al. (2016) further strengthen the latter interpretation, by demonstrating a diffuse stellar envelope that could possibly indicate that the GC is a stripped dSph nucleus.

We find a modest metallicity spread in the cluster $[\mathrm{Fe} / \mathrm{H}]_{\mathrm{NLTE}}=-1.47 \pm 0.06 \mathrm{dex}$. Our LTE result $[\mathrm{Fe} / \mathrm{H}]_{\mathrm{LTE}}=$ $-1.54 \pm 0.06 \mathrm{dex}$ is in good agreement with the previous measurements, in particular with Lardo et al. (2016), who derive $[\mathrm{Fe} / \mathrm{H}]=-1.50 \pm 0.05 \mathrm{dex}$ for the metal-poor component, using Fe II lines. Yong et al. (2014) report three groups with $[\mathrm{Fe} / \mathrm{H}]$ ranging from $-1.66 \pm 0.06 \mathrm{dex}$ to $-1.02 \pm 0.06 \mathrm{dex}$, as derived from the Fe II lines. It should be noted, however, that Lardo et al. (2016) suggest that the metal-rich component may not constitute more than 1

\subsubsection{NGC 6752}

NGC 6752 belongs to the benchmark globular clusters in our Galaxy. Its proximity, $d_{\odot}$ of $4.0 \mathrm{kpc}$ (Harris 1996, 2010 edition), allows a detailed spectroscopic and photometric analysis of the cluster members. The cluster has been extensively observed with the VLT (e.g. Carretta et al. 2007; Gruyters et al. 2014; Lee 2018) and with HST (e.g. Ross et al. 2013; Gruyters et al. 2017; Milone et al. 2019). In particular, deep narrow-band photometric observations have been essential to probe the substructure of this system, with multiple stellar populations identified on the RGB and MS (Dotter et al. 2015; Lee 2018; Milone et al. 2019).

A detailed chemical analysis of the cluster members was presented in different studies. The analysis of high-resolution UVES spectra of 38 RGB stars in NGC 6752 by Yong et al. (2005) showed a prominent $\alpha$-enhancement at $[\mathrm{Mg} / \mathrm{Fe}]=0.47 \pm$ $0.06 \mathrm{dex}$, and the iron abundances of $[\mathrm{Fe} / \mathrm{H}]=-1.56 \pm 0.10 \mathrm{dex}$. Both of these estimates are fully consistent with our LTE results of $[\mathrm{Fe} / \mathrm{H}]_{\mathrm{LTE}}=-1.56 \pm 0.07 \mathrm{dex}$ and $[\mathrm{Mg} / \mathrm{Fe}]_{\mathrm{LTE}}=0.35 \pm$ 0.11 dex. Furthermore, their LTE estimate of $\mathrm{Ti}$ abundance, $[\mathrm{Ti} / \mathrm{Fe}]=0.14 \pm 0.14 \mathrm{dex}$, is consistent with our LTE value, $[\mathrm{Ti} / \mathrm{Fe}]_{\text {LTE }}=0.23 \pm 0.07 \mathrm{dex}$. Our sample is larger than that of Yong et al. (2005) and comprises 110 stars at the base of the RGB, which may account for minor differences between our and their results. On the other hand, our somewhat larger dispersion in abundance ratios is probably not an artefact, as large intra-cluster abundance spreads have also been reported by Yong et al. (2013) from the analysis of high-resolution spectra of RGB stars. Our NLTE estimates are slightly different, but they follow the general trends identified for other metal-poor clusters. The NLTE metallicity and slightly higher, $[\mathrm{Fe} / \mathrm{H}]_{\mathrm{NLTE}}=$ $-1.48 \pm 0.06 \mathrm{dex}$, whereas the NLTE $[\mathrm{Mg} / \mathrm{Fe}]$ ratio is correspondingly lower, $[\mathrm{Mg} / \mathrm{Fe}]_{\mathrm{NLTE}}=0.20 \pm 0.09 \mathrm{dex}$.

\subsubsection{NGC 1904 (M79)}

NGC 1904 is a metal-poor globular cluster at $d_{\odot}=12.9 \mathrm{kpc}$ and $6.3 \mathrm{kpc}$ below the Galactic plane (Harris 1996, 2010 edition). Kains et al. (2012) employed variable stars to determine an accurate distance to the cluster, $13.4 \pm 0.4 \mathrm{kpc}$. The age of the system is $14.1 \pm 2.1 \mathrm{Gyr}$ (Li \& Deng 2018). Similar to NGC 1851, the outskirts of NGC 1904 reveal prominent streams signifying its possible accretion origin (Carballo-Bello et al. 2018; Shipp et al. 2018).

Our NLTE metallicity of the cluster is $[\mathrm{Fe} / \mathrm{H}]_{\mathrm{NLTE}}=-1.51 \pm$ 0.05 dex. This is consistent, modulo the LTE - NLTE difference of -0.07 dex, with the value reported by Carretta et al. (2009), $[\mathrm{Fe} / \mathrm{H}]=-1.58 \pm 0.03 \mathrm{dex}$. Also their LTE Mg abundance, $[\mathrm{Mg} / \mathrm{Fe}]=0.28 \pm 0.06 \mathrm{dex}$, is in good agreement with our LTE value of $[\mathrm{Mg} / \mathrm{Fe}]_{\mathrm{LTE}}=0.31 \pm 0.11 \mathrm{dex}$. Our NLTE estimate is $[\mathrm{Mg} / \mathrm{Fe}]_{\mathrm{NLTE}}=0.16 \pm 0.09 \mathrm{dex}$, which is lower than the LTE value. The cluster is also enriched in $[\mathrm{Ti} / \mathrm{Fe}]$. We find $[\mathrm{Ti} / \mathrm{Fe}]_{\mathrm{NLTE}}=0.21 \pm 0.08 \mathrm{dex}$ and $[\mathrm{Ti} / \mathrm{Fe}]_{\mathrm{LTE}}=0.24 \pm 0.09 \mathrm{dex}$, and the latter is consistent with the LTE results obtained by Fabbian et al. (2005), $[\mathrm{Ti} / \mathrm{Fe}]=0.31 \pm 0.15 \mathrm{dex}$.

\subsubsection{NGC 4833}

The cluster is arguably one of the oldest systems in the Milky Way, with the age of 13.5 Gyr (Wagner-Kaiser et al. 2017). Its location at $d_{\odot}=6.6 \mathrm{kpc}, \sim 1 \mathrm{kpc}$ away from the disk plane (Harris 1996, 2010 edition) and orbital eccentricity are consistent with the cluster being a part of the inner halo system Carretta et al. (2010). The cluster is thought to host multiple populations (Carretta et al. 2014), based on chemical signatures.

A detailed spectroscopic analysis of the cluster has been performed by several groups. Carretta et al. (2014) employed UVES and Giraffe spectra of 78 stars to determine the abundances of 20 elements from $\mathrm{Na}$ to $\mathrm{Nd}$. They obtained relatively small 
dispersions for the majority of elements, including Fe. In contrast, they also found very pronounced $\mathrm{Na}-\mathrm{O}$ and $\mathrm{Mg}-\mathrm{Na}$ anticorrelations and a large intra-cluster variation in the abundances of light elements. Specifically, the $[\mathrm{Mg} / \mathrm{Fe}]$ abundance ratios in the cluster range from slightly sub-solar, $[\mathrm{Mg} / \mathrm{Fe}] \sim-0.05 \mathrm{dex}$, to highly super-solar values, $[\mathrm{Mg} / \mathrm{Fe}] \gtrsim 0.7 \mathrm{dex}$. Another highresolution study of the cluster was presented by Roederer \& Thompson (2015), who obtained high S/N spectra with the MIKE spectrograph at the Magellan II telescope. Their estimates of elemental abundances are somewhat different from Carretta et al. (2014). In particular, they report $[\mathrm{Fe} / \mathrm{H}]=-2.25 \pm 0.02 \mathrm{dex}$ from the neutral $\mathrm{Fe}$ lines, and $[\mathrm{Fe} / \mathrm{H}]=-2.19 \pm 0.01 \mathrm{dex}$ from the ionised Fe lines, attributing the differences with respect to Carretta et al. (2014) to the technical aspects of the analysis, such as the linelist and the solar reference abundances. In terms of abundance inhomogeneities and correlations, their study is consistent with Carretta et al. (2014), with pronounced star-to-star variations in the light elements and signatures of bimodality in $\mathrm{Na}, \mathrm{Al}$, and $\mathrm{Mg}$.

Our LTE estimates of metallicity and abundance ratios are consistent with the literature estimates. In particular, we find $[\mathrm{Fe} / \mathrm{H}]_{\text {LTE }}=-2.08 \pm 0.08 \mathrm{dex}$ and $[\mathrm{Mg} / \mathrm{Fe}]_{\text {LTE }}=0.36 \pm 0.20 \mathrm{dex}$, which can be compared to $[\mathrm{Fe} / \mathrm{H}]=-2.04 \pm 0.02 \mathrm{dex}$ and $[\mathrm{Mg} / \mathrm{Fe}]=0.36 \pm 0.15$ dex derived by Carretta et al. (2014) from the Giraffe spectra. We also confirm that there is negligible internal dispersion in $\mathrm{Ti}$ abundances, with $[\mathrm{Ti} / \mathrm{Fe}]_{\mathrm{LTE}}$ of $0.24 \pm 0.07 \mathrm{dex}$, consistent with Carretta et al. (2014) estimate of $[\mathrm{Ti} / \mathrm{Fe}]=0.17 \pm 0.02 \mathrm{dex}$. On the other hand, our NLTE abundances are considerably different. For Fe, we infer $[\mathrm{Fe} / \mathrm{H}]_{\text {NLTE }}=-1.88 \pm 0.06 \mathrm{dex}$, which is higher compared to $[\mathrm{Fe} / \mathrm{H}]_{\text {LTE }}=-2.08 \pm 0.08 \mathrm{dex}$. Also, the $[\mathrm{Mg} / \mathrm{Fe}]$ ratios are much lower, $[\mathrm{Mg} / \mathrm{Fe}]_{\mathrm{NLTE}}=0.18 \pm 0.17 \mathrm{dex}$, with the abundances in the individual stars ranging from -0.03 to $0.70 \mathrm{dex}$. The NLTE Ti abundances are only slightly higher compared to the LTE estimates, $[\mathrm{Ti} / \mathrm{Fe}]_{\mathrm{NLTE}}=0.22 \pm 0.06 \mathrm{dex}$.

\subsubsection{NGC 4372}

Remarkable for its strong chemical peculiarities, NGC 4372 is nonetheless a rather typical GC system. It is metal-poor and nearby cluster, with an age of $12.5 \mathrm{Gyr}$ (Kruijssen et al. 2019), at a distance of $5.8 \mathrm{kpc}$ and $1.0 \mathrm{kpc}$ below the Galactic plane (Harris 1996, 2010 edition). The cluster reveals a signficant dispersion in $\mathrm{Na}, \mathrm{Mg}, \mathrm{Al}$, and $\mathrm{O}$, a $\mathrm{Na}-\mathrm{O}$ anti-correlation, and, possibly, an Al-Mg anti-correlation (San Roman et al. 2015).

Our average NLTE metallicity of stars in NGC 4372 is $-2.07 \pm 0.06 \mathrm{dex}$. Our LTE metallicity is much lower, $[\mathrm{Fe} / \mathrm{H}]_{\mathrm{LTE}}=-2.33 \pm 0.08 \mathrm{dex}$, following the general trend for all metal-poor clusters seen in Fig. 3. Comparing the latter estimate with the literature, we find a satisfactory agreement with a comprehensive study by San Roman et al. (2015), which is also based on the spectra acquired within the GaiaESO survey. Their estimate of $[\mathrm{Fe} / \mathrm{H}]$ is $-2.23 \pm 0.10 \mathrm{dex}^{11}$, consistent with our results within the combined uncertainties of the both estimates. Also the value from Carretta et al. (2009), $[\mathrm{Fe} / \mathrm{H}]=-2.19 \pm 0.08 \mathrm{dex}$, is somewhat higher than our LTE metallicity. The detailed abundance ratios of our study are also in agreement with those measured by San Roman et al. (2015). We obtain $[\mathrm{Mg} / \mathrm{Fe}]_{\mathrm{LTE}}=0.51 \pm 0.09 \mathrm{dex}$ and $[\mathrm{Ti} / \mathrm{Fe}]_{\mathrm{LTE}}=$ $0.22 \pm 0.07$ dex in LTE, whereas San Roman et al. (2015) derive $[\mathrm{Mg} / \mathrm{Fe}]=0.44 \pm 0.07 \mathrm{dex}$ and $[\mathrm{Ti} / \mathrm{Fe}]=0.31 \pm 0.03 \mathrm{dex}$.

\footnotetext{
${ }^{11}$ Note that this value depends on whether large outliers are included or not.
}

Our NLTE estimates are $[\mathrm{Mg} / \mathrm{Fe}]_{\mathrm{NLTE}}=0.31 \pm 0.07 \mathrm{dex}$ and $[\mathrm{Ti} / \mathrm{Fe}]_{\mathrm{NLTE}}=0.20 \pm 0.06 \mathrm{dex}$.

\subsubsection{M15 (NGC 7078)}

Similar to NGC 1904 and NGC 4833, M 15 represents one of the oldest and metal-poor systems in the Galactic halo at a distance $d_{\odot}=10.4 \mathrm{kpc}$ and $4.8 \mathrm{kpc}$ below the Galactic plane (Harris 1996, 2010 edition). Several studies report multiple stellar populations in the cluster (Larsen et al. 2015; Nardiello et al. 2018; Bonatto et al. 2019).

M 15 has the lowest metallicity in our sample and shows the largest NLTE effects: $[\mathrm{Fe} / \mathrm{H}]_{\mathrm{NLTE}}=-2.28 \pm 0.06 \mathrm{dex}$, but $[\mathrm{Fe} / \mathrm{H}]_{\mathrm{LTE}}=-2.58 \pm 0.07 \mathrm{dex}$. Our LTE estimate compares favourably well with Sobeck et al. (2011), who derived $[\mathrm{Fe} / \mathrm{H}]=-2.62 \pm 0.08 \mathrm{dex}^{12}$ from the analysis of high-resolution spectra of several RGB and RHB stars in the cluster collected with the HIRES spectrograph at the Keck telescope. Worley et al. (2013) report $[\mathrm{Fe} / \mathrm{H}]$ in the range from -2.4 to -2.3 dex with an uncertainty of $0.1 \mathrm{dex}$, which is closer to the estimate of $[\mathrm{Fe} / \mathrm{H}]=-2.37$ dex derived by Letarte et al. (2006) and $[\mathrm{Fe} / \mathrm{H}]=-2.32$ dex by Carretta et al. (2009). Our average LTE abundances of $\mathrm{Mg}$ is $[\mathrm{Mg} / \mathrm{Fe}]_{\mathrm{LTE}}=0.36 \pm 0.23 \mathrm{dex}$, with the star-to-star variation in the range from -0.26 to $0.66 \mathrm{dex}$. This is consistent with Carretta et al. (2009), within the uncertainties, and also with the abundances derived by Sobeck et al. (2011), who measured $[\mathrm{Mg} / \mathrm{Fe}]$ ratios from -0.01 to $0.6 \mathrm{dex}$. In contrast, the cluster stars exhibit very tight $[\mathrm{Ti} / \mathrm{Fe}]$ ratios with the mean of $[\mathrm{Ti} / \mathrm{Fe}]_{\mathrm{LTE}}=0.19 \pm 0.05 \mathrm{dex}$. Our NLTE results for $\mathrm{Mg}$ are much lower than the LTE ones, $[\mathrm{Mg} / \mathrm{Fe}]_{\mathrm{NLTE}}=0.22 \pm 0.19 \mathrm{dex}$, whereas the NLTE $\mathrm{Ti}$ abundances are nearly consistent with LTE, $[\mathrm{Ti} / \mathrm{Fe}]_{\mathrm{NLTE}}=0.21 \pm 0.05 \mathrm{dex}$.

\subsection{Comparison with Milky Way field stars}

It is useful to combine our chemical characterisation of the clusters with their kinematics, in order to compare our results with Galactic field stars. We employ the kinematic selection criteria from Bensby et al. (2014) to assign Galactic population membership to the clusters (see Appendix C). The Toomre diagram for the clusters and field stars is shown in Fig. 9.

In Figs. 10 and 11, we overlay our LTE and NLTE abundance ratios in the clusters with the literature measurements in the Galactic field stars. The field sample is taken from Bensby et al. (2014) and Bergemann et al. (2017b). The former dataset represents populations in the solar neighbourhood and has a large coverage in metallicity, $-2.7 \lesssim[\mathrm{Fe} / \mathrm{H}] \lesssim 0.5$. The $\mathrm{Fe}$ abundances were derived in NLTE, while $\mathrm{Mg}$ and $\mathrm{Ti}$ were derived in LTE analysis. The dataset Bergemann et al. (2017b) lacks a thin disk component, $[\mathrm{Fe} / \mathrm{H}]>-0.5$, but contains a significant fraction of the thick disk and halo stars. The study provides LTE and NLTE estimates of $[\mathrm{Fe} / \mathrm{H}]$ and $[\mathrm{Mg} / \mathrm{Fe}]$ derived using $1 \mathrm{D}$ and $\langle 3 \mathrm{D}\rangle$ atmospheric models. For consistency with our 1D analysis, we use their 1D LTE and 1D NLTE results.

There are several important results, which stand out by comparing our LTE and NLTE measurements in clusters against Galactic field stars. Firstly, our LTE abundances in GCs trace the Galactic field population remarkably well, at least as long as LTE field distributions are employed for the comparison. This supports the conclusions drawn by Pritzl et al. (2005). NGC 3532 and NGC 2243, the two metal-rich clusters with disk-like 12 We recompute value using the mean of all measurements from nine
RGB and RHB stars. 


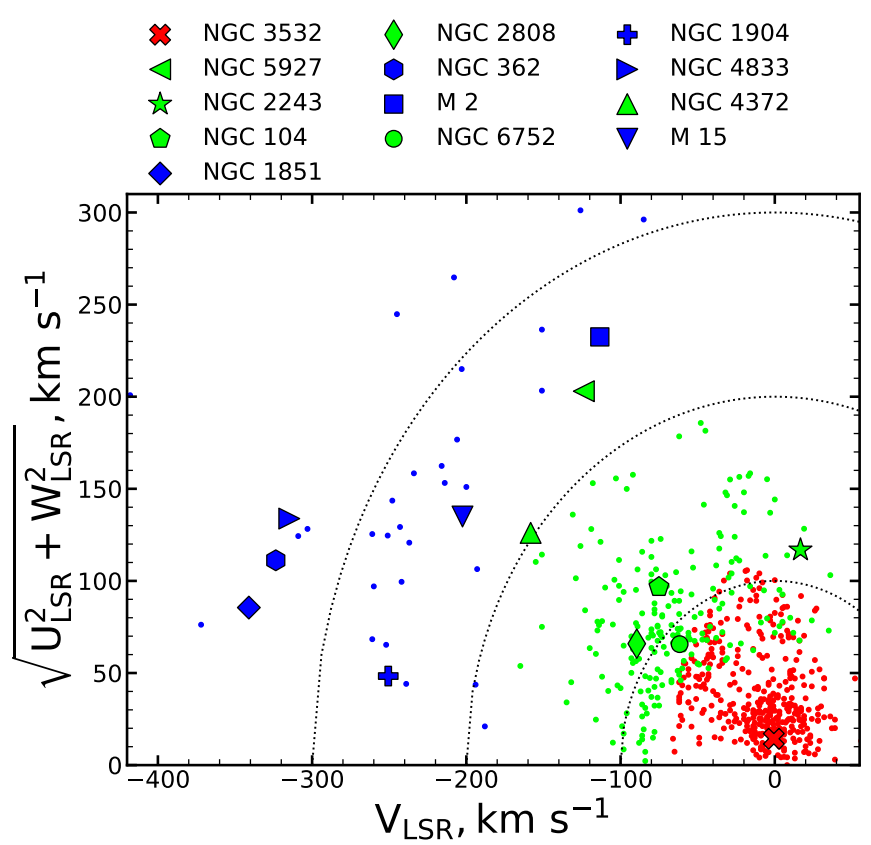

Fig. 9. Toomre diagram for clusters and Bensby et al. (2014) field stars. The thin disk population is shown in red colour, the thick disk population in green colour, and the halo population in blue colour. The isolines for total velocity $V_{\mathrm{tot}}=\sqrt{U_{\mathrm{LSR}}^{2}+V_{\mathrm{LSR}}^{2}+W_{\mathrm{LSR}}^{2}}=100,200,300 \mathrm{~km} \mathrm{~s}^{-1}$ are shown as dotted lines. For the details of population assignment see Appendix C.

kinematic properties, occupy the chemical locus of the thin disk. The metal-poor globular clusters trace the thick disk and the halo. Despite a difference of two orders of magnitude in metallicity, all metal-poor GCs follow very tight trends of the average $[\mathrm{Mg} / \mathrm{Fe}]$ and $[\mathrm{Ti} / \mathrm{Fe}]$ with $[\mathrm{Fe} / \mathrm{H}]$. In particular, all of them occupy the locus situated at $[\mathrm{Ti} / \mathrm{Fe}] \approx 0.25 \mathrm{dex}$ with small dispersion. On the other hand, the intra-cluster dispersions of $[\mathrm{Mg} / \mathrm{Fe}]$ increase substantially. This is not unexpected and has been extensively discussed in the literature (Gratton et al. 2004; Carretta et al. 2014; Carretta 2014). The large variation of $\mathrm{Mg}$ abundances is usually attributed to the nuclear processing associated with high temperature hydrogen burning and multiple star formation episodes. In such a scenario first generation massive stars evolve fast, converting their $\mathrm{Mg}$ into Al. Second generation stars, formed from the material of first generation stars, are depleted in $\mathrm{Mg}$ and enriched in Al. The absence of any noticeable dispersion in $[\mathrm{Ti} / \mathrm{Fe}]$ in all GCs corroborates this interpretation.

Notwithstanding the good agreement of our LTE results with earlier LTE studies, we find important differences between LTE and NLTE results (Fig. 11), which impact the astrophysical interpretation of the results. When comparing our NLTE abundances for globular clusters with the NLTE abundances of field stars, only two metal-rich clusters with the thick disk kinematics (NGC 104 and NGC 5927) and the metal-poor cluster NGC 4372 appear to be consistent with the field stars. All other metal-poor clusters are systematically depleted in $[\mathrm{Mg} / \mathrm{Fe}]$ relative to the metal-poor disk and the halo. This may imply that the metalpoor clusters were not formed in-situ, but were accreted from disrupted dwarf satellite galaxies.

\section{Conclusions}

In this work, we employ non-LTE radiative transfer models and the Payne code to determine chemical abundances for 13 stellar clusters in the Milky Way. The observed spectra are taken from the third public data release of the Gaia-ESO survey, and we focus on the $R \sim 19800$ spectra taken with the Giraffe instrument. The NLTE synthetic spectra are computed using the model atoms presented in earlier works (Bergemann \& Gehren 2008; Bergemann 2011; Bergemann et al. 2012, 2017a). The Payne code is used to interpolate in the grids of synthetic spectra to maximise the efficiency of the analysis, where we simultaneously fit for all spectral parameters, exploring more information from the full spectrum. The spectral grids are computed at random nodes in stellar parameter space and a $\chi^{2}$ minimisation is employed to find the best-fit stellar parameters and chemical abundances by comparing the models with the observations.

We validate our method and the models on the Gaia-ESO benchmark stars, for which stellar parameters are well constrained by parallaxes, asteroseismology, and interferometric angular diameter measurements. The calibration sample includes 19 main-sequence dwarfs, subgiants, and red giants in the $[\mathrm{Fe} / \mathrm{H}]$ range from -2.5 to 0.3 dex with spectra taken at different exposure times spanning the $\mathrm{S} / \mathrm{N}$ range of $100-2800 \AA^{-1}$. We find a very good agreement between our NLTE spectroscopic results and the independently determined stellar parameters. The residuals are within $-29 \pm 88 \mathrm{~K}$ in $T_{\text {eff }}, 0.09 \pm 0.16 \mathrm{dex}$ in $\log (g)$, and $0.02 \pm 0.09 \mathrm{dex}$ in $[\mathrm{Fe} / \mathrm{H}]$. The analysis of repeat observations of the same stars indicates the absence of a systematic bias or correlation of the abundance error with the quality the spectra within the full range of $\mathrm{S} / \mathrm{N}$ probed in this work.

We compute stellar parameters and abundances for 742 stars in two open clusters and 11 globular clusters in the Milky Way galaxy. The results are provided in Table A.5 and are archived electronically on CDS. The typical S/N of the spectra is $200 \AA^{-1}$. We find that spectroscopic estimates of stellar parameters $\left(T_{\text {eff }}, \log g\right.$, and $\left.[\mathrm{Fe} / \mathrm{H}]\right)$ agree with evolutionary expectations, based on isochrones. However, different isochrones are needed to match the LTE and NLTE data. At low metallicity, the difference between LTE and NLTE parameters is significant, confirming earlier studies (i.e. Bergemann et al. 2012; Lind et al. 2012; Ruchti et al. 2013). The systematic error of LTE increases in proportionality with decreasing metallicity, and amounts to $300 \mathrm{~K}$ in $T_{\text {eff }}, 0.6$ dex in $\log g$, and $0.3 \mathrm{dex}$ in $[\mathrm{Fe} / \mathrm{H}]$ for the RGB stars with $[\mathrm{Fe} / \mathrm{H}]_{\mathrm{NLTE}}=-2.3$. The $[\mathrm{Mg} / \mathrm{Fe}]$ abundance ratios are typically lower in NLTE compared to LTE. Our abundances show no significant trends with stellar parameters, supporting their relative accuracy.

Our results for the Galactic open and globular clusters can be summarised as follows:

- NGC 3532, a young metal-rich open cluster, is consistent in its chemical abundance pattern and its kinematics with the Galactic thin disk. The cluster is slightly depleted in $\mathrm{Mg}$ compared to the solar neighbourhood, although the difference is generally within the uncertainties of the abundance measurements.

- NGC 2243, a relatively old open cluster lies on the metalpoor end of the thin disk track, and shows a noticeable dispersion in $[\mathrm{Fe} / \mathrm{H}],[\mathrm{Mg} / \mathrm{Fe}]$, and $[\mathrm{Ti} / \mathrm{Fe}]$ ratios contrasting with the tight chemical patterns in the field stars. This is the only cluster in our sample that is represented by mainsequence and TO stars, and this spread likely has an astrophysical origin. In particular, the pronounced dip in $[\mathrm{Fe} / \mathrm{H}]$ at the TO signifies the action of atomic diffusion consistent with depletion predicted by detailed stellar evolution models.

- Two metal-rich clusters with thick disk like kinematics NGC 104 and NGC 5927 are also very similar to the thick disk in their abundance ratios of $[\mathrm{Mg} / \mathrm{Fe}]$ and $[\mathrm{Ti} / \mathrm{Fe}]$. They 

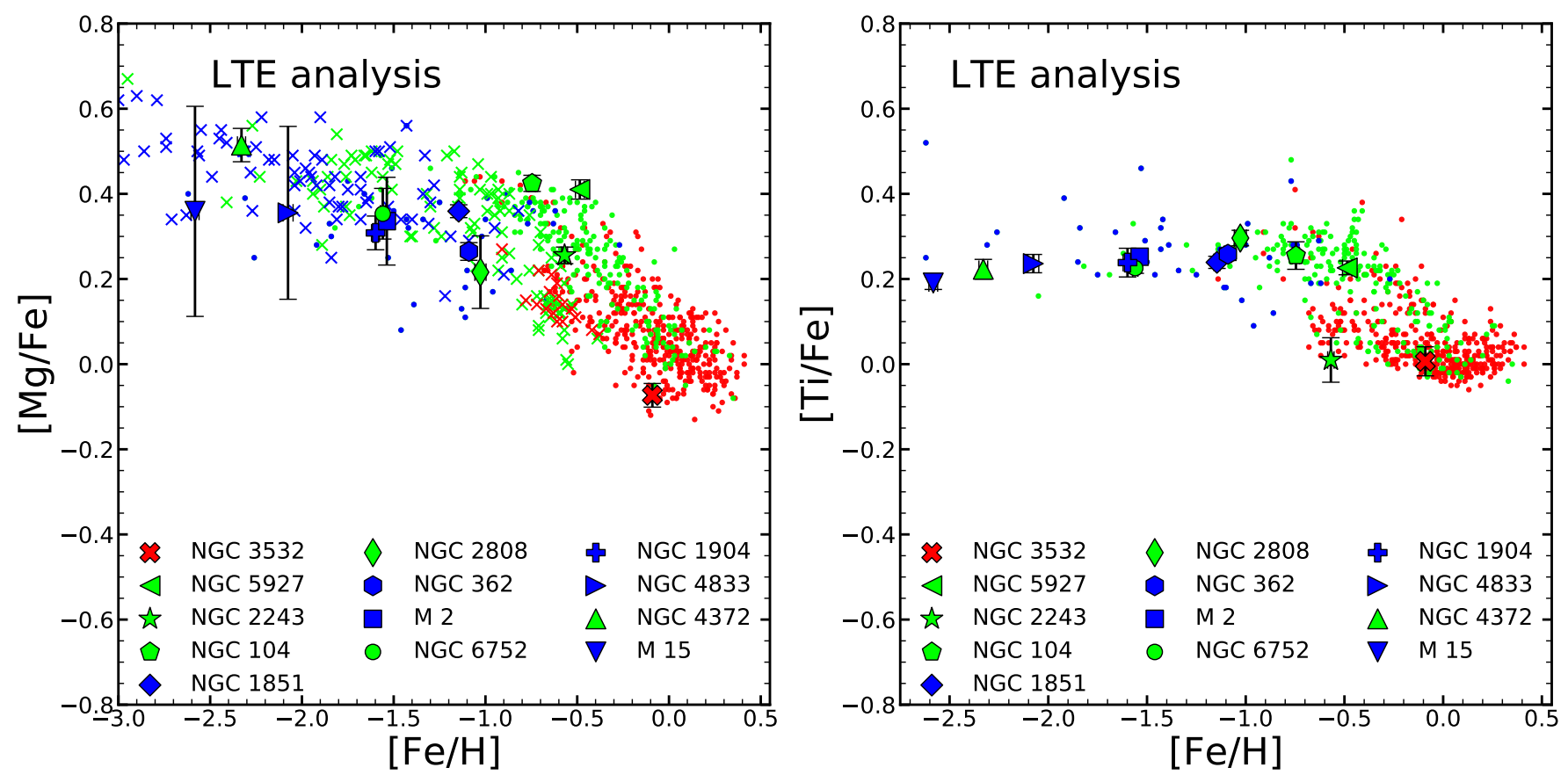

Fig. 10. Mean LTE metallicities and $[\mathrm{Mg} / \mathrm{Fe}]$ and $[\mathrm{Ti} / \mathrm{Fe}]$ abundance ratios for all clusters and for Milky Way field stars from Bensby et al. (2014) (NLTE $[\mathrm{Fe} / \mathrm{H}]$, LTE $[\mathrm{Mg} / \mathrm{Fe}]$ and LTE $[\mathrm{Ti} / \mathrm{Fe}]$ - small dots) and Bergemann et al. (2017b) (1D LTE results - small crosses). Error bars represent the $1 \sigma$ intra-cluster abundance variations. Colours are the same as in Fig. 9.
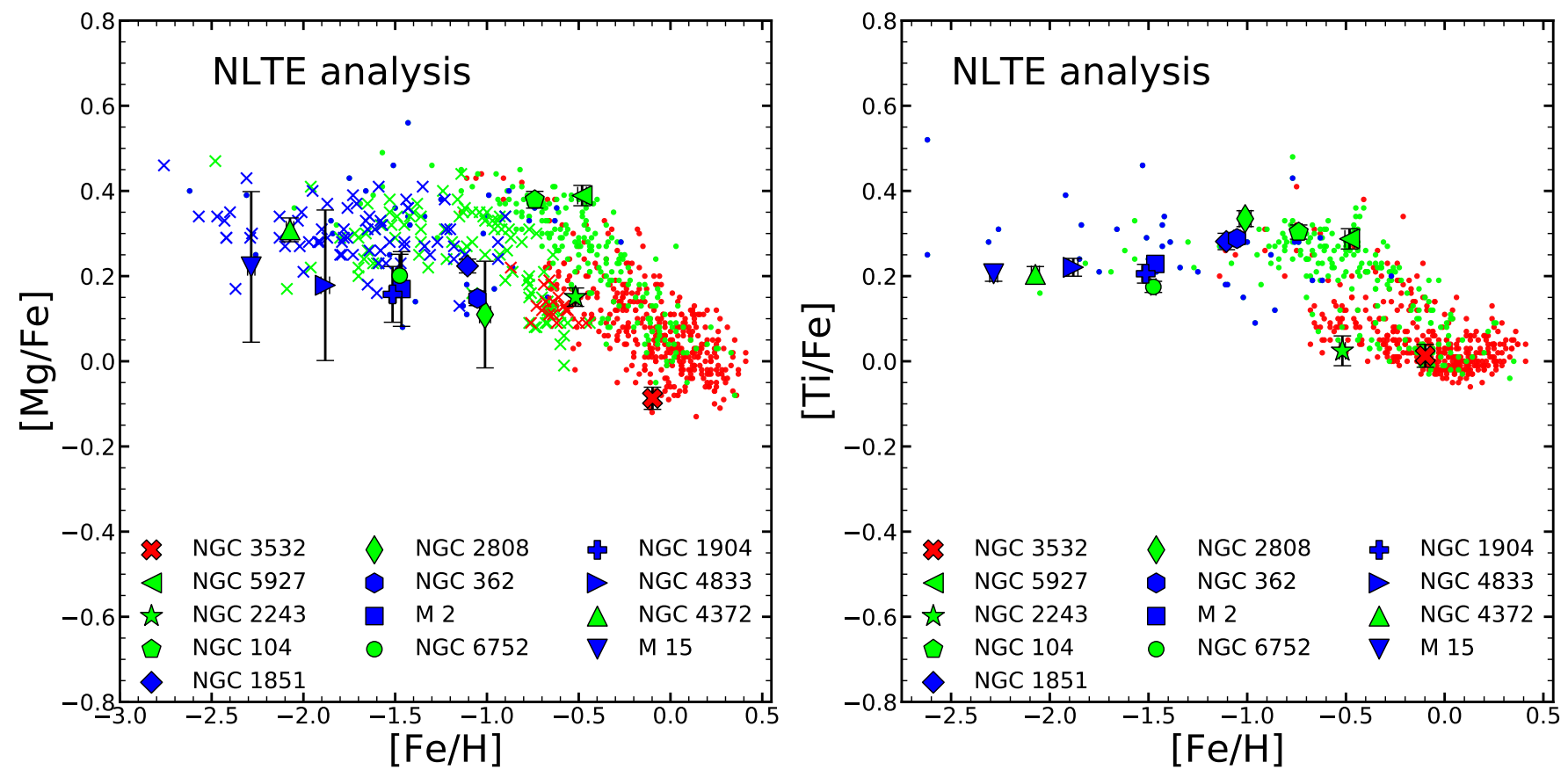

Fig. 11. Mean NLTE metallicities and $[\mathrm{Mg} / \mathrm{Fe}]$ and $[\mathrm{Ti} / \mathrm{Fe}]$ abundance ratios for all clusters and for Milky Way field stars from Bensby et al. (2014) (NLTE $[\mathrm{Fe} / \mathrm{H}]$, LTE $[\mathrm{Mg} / \mathrm{Fe}]$ and LTE [Ti/Fe] - small dots) and Bergemann et al. (2017b) (1D NLTE results - small crosses). Error bars represent the $1 \sigma$ intra-cluster abundance variations. Colours are the same as in Fig. 9.

show small dispersions in all elements $\lesssim 0.06$ dex, which are much smaller then the typical systematic uncertainties of our measurements, and are consistent with being chemically homogeneous populations.

- The metal-poor clusters NGC 2808 and NGC 6752, despite being kinematically similar to the thick disk, appear to be depleted in $[\mathrm{Mg} / \mathrm{Fe}]$ compared to the field stars, based on
NLTE analysis. On the other hand, their $[\mathrm{Ti} / \mathrm{Fe}]$ ratios are representative of the halo clusters.

- NLTE analysis suggests that the majority of metal-poor clusters with $[\mathrm{Fe} / \mathrm{H}]<-1$ dex and halo-like kinematics, show a prominent, $\sim 0.15 \mathrm{dex}$, depletion of $[\mathrm{Mg} / \mathrm{Fe}]$ compared to field stars of the same metallicity. This may indicate their ex situ formation history. 
- NGC 2808 and NGC 1851 exhibit remarkably similar chemical abundance patterns and overlap in metallicity that reinforces the evidence for their common origin proposed in the literature.

- Large intra-cluster spreads in $[\mathrm{Mg} / \mathrm{Fe}]$, compared to the field population, are seen in the clusters M 2, NGC 2808, NGC 4833 and M 15, corroborating with the long-postulated scenario that globular clusters have undergone multiple episodes of star formation and self-enrichment. On the other hand, the clusters are homogeneous in $[\mathrm{Ti} / \mathrm{Fe}]$.

- The metal-poor globular cluster NGC 4372 stands out in comparison with the other globular clusters with a similar metallicity. Its $[\mathrm{Mg} / \mathrm{Fe}]$ spread is relatively small, consistent with the study by San Roman et al. (2015). Given our standard abundance uncertainties of $\sim 0.1$ dex, which exceed the intra-cluster dispersion, the cluster is homogeneous in $[\mathrm{Fe} / \mathrm{H}],[\mathrm{Mg} / \mathrm{Fe}]$ and $[\mathrm{Ti} / \mathrm{Fe}]$.

- For M 15 and NGC 4833, which are the most metal-poor clusters in our sample, we find strong evidence for a multimodality in $[\mathrm{Mg} / \mathrm{Fe}]$. However, our samples are too small to draw statistically robust conclusions on whether these clusters host two or more sub-populations.

The combination of NLTE models and the Payne is a powerful tool for homogeneous analysis of the stellar parameters and chemical abundances. Our results for a large sample of stars in wide range of metallicity suggests that NLTE effects are significant for metal-poor regime $([\mathrm{Fe} / \mathrm{H}]<-1)$ and should be always taken into account.

Acknowledgements. We thank Nikolay Kacharov, Diane Feuillet and David Hogg for valuable discussions. We thank anonymous referee for useful suggestions. Based on data products from observations made with ESO Telescopes at the La Silla Paranal Observatory under programme ID 188.B-3002. This work has made use of data from the European Space Agency (ESA) mission Gaia (https://www.cosmos.esa.int/gaia), processed by the Gaia Data Processing and Analysis Consortium (DPAC, https://www.cosmos.esa.int/ web/gaia/dpac/consortium). Funding for the DPAC has been provided by national institutions, in particular the institutions participating in the Gaia Multilateral Agreement. This research has made use of the WEBDA database, operated at the Department of Theoretical Physics and Astrophysics of the Masaryk University. We acknowledge support by the Collaborative Research centre SFB 881 (Heidelberg University) of the Deutsche Forschungsgemeinschaft (DFG, German Research Foundation). YST is supported by the NASA Hubble Fellowship grant HST-HF2-51425.001 awarded by the Space Telescope Science Institute.

\section{References}

Amarsi, A. M., Lind, K., Asplund, M., Barklem, P. S., \& Collet, R. 2016, MNRAS, 463, 1518

Amarsi, A. M., Barklem, P. S., Asplund, M., Collet, R., \& Zatsarinny, O. 2018, A\&A, 616, A89

Anderson, J., Piotto, G., King, I. R., Bedin, L. R., \& Guhathakurta, P. 2009, ApJ, 697, L58

Anthony-Twarog, B. J., Atwell, J., \& Twarog, B. A. 2005, AJ, 129, 872

Astropy Collaboration (Robitaille, T. P., et al.) 2013, A\&A, 558, A33

Auer, L. H., \& Mihalas, D. 1969, ApJ, 156, 157

Barklem, P. S. 2016, A\&ARv, 24, 9

Barklem, P. S. 2018, A\&A, 612, A90

Barklem, P. S., Osorio, Y., Fursa, D. V., et al. 2017, A\&A, 606, A11

Bastian, N., \& Lardo, C. 2018, ARA\&A, 56, 83

Baumgardt, H., \& Hilker, M. 2018, MNRAS, 478, 1520

Bautista, M. A., Lind, K., \& Bergemann, M. 2017, A\&A, 606, A127

Bekki, K., \& Yong, D. 2012, MNRAS, 419, 2063

Bellazzini, M., Pecci, F. F., Ferraro, F. R., et al. 2001, AJ, 122, 2569

Belyaev, A. K., \& Yakovleva, S. A. 2017, A\&A, 606, A147

Bensby, T., Feltzing, S., \& Oey, M. S. 2014, A\&A, 562, A71

Bergemann, M. 2011, MNRAS, 413, 2184

Bergemann, M., \& Gehren, T. 2008, A\&A, 492, 823

Bergemann, M., Kudritzki, R.-P., Plez, B., et al. 2012, ApJ, 751, 156

Bergemann, M., Collet, R., Amarsi, A. M., et al. 2017a, ApJ, 847, 15
Bergemann, M., Collet, R., Schönrich, R., et al. 2017b, ApJ, 847, 16 Blanco-Cuaresma, S., Soubiran, C., Jofré, P. \& Heiter, U. 2014, A\&A, 566, A98 Bonatto, C., Chies-Santos, A. L., Coelho, P. R. T., et al. 2019, A\&A, 622, A179 Brogaard, K., VandenBerg, D. A., Bedin, L. R., et al. 2017, MNRAS, 468, 645 Buder, S., Lind, K., Ness, M. K., et al. 2019, A\&A, 624, A19

Butler, K., \& Giddings, J. 1985, Newsletter on the Analysis of Astronomical Spectra No. 9 (London: University of London)

Campos, F., Pelisoli, I., Kamann, S., et al. 2018, MNRAS, 481, 4397

Carballo-Bello, J. A., Martínez-Delgado, D., Navarrete, C., et al. 2018, MNRAS, 474,683

Carretta, E. 2006, AJ, 131, 1766

Carretta, E. 2014, ApJ, 795, L28

Carretta, E. 2015, ApJ, 810, 148

Carretta, E., Bragaglia, A., Gratton, R. G., et al. 2006, A\&A, 450, 523

Carretta, E., Bragaglia, A., Gratton, R. G., Lucatello, S., \& Momany, Y. 2007, A\&A, 464, 927

Carretta, E., Bragaglia, A., Gratton, R., \& Lucatello, S. 2009, A\&A, 505, 139

Carretta, E., Bragaglia, A., Gratton, R. G., et al. 2010, A\&A, 516, A55

Carretta, E., Lucatello, S., Gratton, R. G., Bragaglia, A., \& D’Orazi, V. 2011, A\&A, 533, A69

Carretta, E., Bragaglia, A., Gratton, R. G., et al. 2013, A\&A, 557, A138

Carretta, E., Bragaglia, A., Gratton, R. G., et al. 2014, A\&A, 564, A60

Casetti-Dinescu, D. I., Girard, T. M., Herrera, D., et al. 2007, AJ, 134, 195

Casey, A. R., Hawkins, K., Hogg, D. W., et al. 2017, ApJ, 840, 59

Černiauskas, A., Kučinskas, A., Klevas, J., et al. 2017, A\&A, 604, A35

Chen, S., Richer, H., Caiazzo, I., \& Heyl, J. 2018, ApJ, 867, 132

Clem, J. L., Landolt, A. U., Hoard, D. W., \& Wachter, S. 2011, AJ, 141, 115

Conrad, C., Scholz, R.-D., Kharchenko, N. V., et al. 2014, A\&A, 562, A54

Cordero, M. J., Pilachowski, C. A., Johnson, C. I., et al. 2014, ApJ, 780, 94

Creevey, O., Grundahl, F., Thévenin, F., et al. 2019, A\&A, 625, A33

Cummings, J. D., Geisler, D., \& Villanova, S. 2017, AJ, 153, 192

Cunha, K., Frinchaboy, P. M., Souto, D., et al. 2016, Astron. Nachr., 337, 922

Damiani, F., Bonito, R., Magrini, L., et al. 2016, A\&A, 591, A74

D’Antona, F., Bellazzini, M., Caloi, V., et al. 2005, ApJ, 631, 868

Deal, M., Alecian, G., Lebreton, Y., et al. 2018, A\&A, 618, A10

De Silva, G. M., Freeman, K. C., Bland-Hawthorn, J., et al. 2015, MNRAS, 449, 2604

Dobbie, P. D., Napiwotzki, R., Burleigh, M. R., et al. 2009, MNRAS, 395, 2248

Dobbie, P. D., Day-Jones, A., Williams, K. A., et al. 2012, MNRAS, 423, 2815

Dobrovolskas, V., Kučinskas, A., Bonifacio, P., et al. 2014, A\&A, 565, A121

D'Orazi, V., Gratton, R. G., Angelou, G. C., et al. 2015, MNRAS, 449, 4038

Dotter, A., Sarajedini, A., Anderson, J., et al. 2010, ApJ, 708, 698

Dotter, A., Ferguson, J. W., Conroy, C., et al. 2015, MNRAS, 446, 1641

Fabbian, D., Recio-Blanco, A., Gratton, R. G., \& Piotto, G. 2005, A\&A, 434, 235

François, P., Pasquini, L., Biazzo, K., Bonifacio, P., \& Palsa, R. 2013, A\&A, 552, A136

Friel, E. D., Janes, K. A., Tavarez, M., et al. 2002, AJ, 124, 2693

Fritzewski, D. J., Barnes, S. A., James, D. J., et al. 2019, A\&A, 622, A110

Fu, X., Bressan, A., Marigo, P., et al. 2018, MNRAS, 476, 496

Gaia Collaboration (Brown, A. G. A., et al.) 2018, A\&A, 616, A1

Gilmore, G., Randich, S., Asplund, M., et al. 2012, The Messenger, 147, 25

Gratton, R. G. 1982, ApJ, 257, 640

Gratton, R. G., \& Contarini, G. 1994, A\&A, 283, 911

Gratton, R., Sneden, C., \& Carretta, E. 2004, ARA\&A, 42, 385

Gratton, R. G., Carretta, E., Bragaglia, A., Lucatello, S., \& D’Orazi, V. 2010, A\&A, 517, A81

Gratton, R. G., Villanova, S., Lucatello, S., et al. 2012, A\&A, 544, A12

Gray, D. F. 2005, The Observation and Analysis of Stellar Photospheres (Cambridge, UK: Cambridge University Press)

Grevesse, N., \& Sauval, A. J. 1998, Space Sci. Rev., 85, 161

Grevesse, N., Asplund, M., \& Sauval, A. J. 2007, Space Sci. Rev., 130, 105

Grupp, F. 2004a, A\&A, 420, 289

Grupp, F. 2004b, A\&A, 426, 309

Gruyters, P., Nordlander, T., \& Korn, A. J. 2014, A\&A, 567, A72

Gruyters, P., Casagrande, L., Milone, A. P., et al. 2017, A\&A, 603, A37

Harris, W. E. 1996, AJ, 112, 1487

Heiter, U., Soubiran, C., Netopil, M., \& Paunzen, E. 2014, A\&A, 561, A93

Jacobson, H. R., Friel, E. D., \& Pilachowski, C. A. 2011, AJ, 141, 58

Jofré, P., Heiter, U., Soubiran, C., et al. 2015, A\&A, 582, A81

Kacharov, N., Bianchini, P., Koch, A., et al. 2014, A\&A, 567, A69

Kains, N., Bramich, D. M., Figuera Jaimes, R., et al. 2012, A\&A, 548, A92

Kaluzny, J., Pych, W., Rucinski, S. M., \& Thompson, I. B. 2006, Act Astron., 56, 237

Karovicova, I., White, T. R., Nordlander, T., et al. 2018, MNRAS, 475, L81

Koch, A., \& McWilliam, A. 2008, AJ, 135, 1551

Kruijssen, J. M. D., Pfeffer, J. L., Reina-Campos, M., Crain, R. A., \& Bastian, N. 2019, MNRAS, 486, 3180 
Kučinskas, A., Dobrovolskas, V., \& Bonifacio, P. 2014, A\&A, 568, L4 Kuzma, P. B., Da Costa, G. S., Mackey, A. D., \& Roderick, T. A. 2016, MNRAS, 461, 3639

Kuzma, P. B., Da Costa, G. S., \& Mackey, A. D. 2018, MNRAS, 473, 2881

Lapenna, E., Mucciarelli, A., Lanzoni, B., et al. 2014, ApJ, 797, 124

Lardo, C., Pancino, E., Mucciarelli, A., \& Milone, A. P. 2012, A\&A, 548, A107

Lardo, C., Pancino, E., Mucciarelli, A., et al. 2013, MNRAS, 433, 1941

Lardo, C., Mucciarelli, A., \& Bastian, N. 2016, MNRAS, 457, 51

Larsen, S. S., Baumgardt, H., Bastian, N., et al. 2015, ApJ, 804, 71

Lee, J.-W. 2018, ApJS, 238, 24

Letarte, B., Hill, V., Jablonka, P., et al. 2006, A\&A, 453, 547

Li, Z., \& Deng, Y. 2018, Ap\&SS, 363, 97

Lim, D., Lee, Y.-W., Pasquato, M., Han, S.-I., \& Roh, D.-G. 2016, ApJ, 832, 99

Lind, K., Bergemann, M., \& Asplund, M. 2012, MNRAS, 427, 50

Majewski, S. R., Schiavon, R. P., Frinchaboy, P. M., et al. 2017, AJ, 154, 94

Marigo, P., Girardi, L., Bressan, A., et al. 2017, ApJ, 835, 77

Marino, A. F., Milone, A. P., Yong, D., et al. 2014, MNRAS, 442, 3044

Marino, A. F., Milone, A. P., Casagrande, L., et al. 2016, MNRAS, 459, 610

Martin, N. F., Ibata, R. A., Bellazzini, M., et al. 2004, MNRAS, 348, 12

Menzies, J. W. 1967, Nature, 214, 689

Milone, A. P., Bedin, L. R., Piotto, G., et al. 2008, ApJ, 673, 241

Milone, A. P., Piotto, G., Bedin, L. R., et al. 2012, ApJ, 744, 58

Milone, A. P., Marino, A. F., Piotto, G., et al. 2015, ApJ, 808, 51

Milone, A. P., Marino, A. F., Bedin, L. R., et al. 2019, MNRAS, 484, 4046

Mura-Guzmán, A., Villanova, S., Muñoz, C., \& Tang, B. 2018, MNRAS, 474, 4541

Nardiello, D., Milone, A. P., Piotto, G., et al. 2018, MNRAS, 477, 2004

Ness, M., Hogg, D. W., Rix, H.-W., Ho, A. Y. Q., \& Zasowski, G. 2015, ApJ, 808,16

Netopil, M. 2017, MNRAS, 469, 3042

Osorio, Y., Barklem, P. S., Lind, K., et al. 2015, A\&A, 579, A53

Pancino, E., Lardo, C., Altavilla, G., et al. 2017a, A\&A, 598, A5

Pancino, E., Romano, D., Tang, B., et al. 2017b, A\&A, 601, A112

Pasquini, L., Avila, G., Blecha, A., et al. 2002, The Messenger, 110, 1

Pehlivan Rhodin, A., Hartman, H., Nilsson, H., \& Jönsson, P. 2017, A\&A, 598, A102

Piatti, A. E., \& Koch, A. 2018, AJ, 867, 8

Piotto, G., Bedin, L. R., Anderson, J., et al. 2007, ApJ, 661, L53

Piotto, G., Milone, A. P., Anderson, J., et al. 2012, ApJ, 760, 39

Pritzl, B. J., Venn, K. A., \& Irwin, M. 2005, AJ, 130, 2140
Randich, S., Gilmore, G., \& Consortium, G.-E. 2013, The Messenger, 154, 47

Recio-Blanco, A., Bijaoui, A., \& de Laverny, P. 2006, MNRAS, 370, 141

Reetz, J. 1991, PhD Thesis, Diplomarbeit, Universität München, Germany Roederer, I. U., \& Thompson, I. B. 2015, MNRAS, 449, 3889

Ross, T. L., Holtzman, J., Anthony-Twarog, B. J., et al. 2013, ArXiv e-prints [arXiv:1310.4553]

Ruchti, G. R., Bergemann, M., Serenelli, A., Casagrande, L., \& Lind, K. 2013, MNRAS, 429, 126

San Roman, I., Muñoz, C., Geisler, D., et al. 2015, A\&A, 579, A6

Santos, N. C., Lovis, C., Melendez, J., et al. 2012, A\&A, 538, A151

Schönrich, R., \& Bergemann, M. 2014, MNRAS, 443, 698

Schönrich, R., Binney, J., \& Dehnen, W. 2010, MNRAS, 403, 1829

Shipp, N., Drlica-Wagner, A., Balbinot, E., et al. 2018, ApJ, 862, 114

Simioni, M., Milone, A. P., Bedin, L. R., et al. 2016, MNRAS, 463, 449

Simpson, J. D., Martell, S. L., \& Navin, C. A. 2017, MNRAS, 465, 1123

Smiljanic, R., Korn, A. J., Bergemann, M., et al. 2014, A\&A, 570, A122

Smith, G. H., \& Langland-Shula, L. E. 2009, PASP, 121, 1054

Smith, G. H., \& Mateo, M. 1990, ApJ, 353, 533

Sobeck, J. S., Kraft, R. P., Sneden, C., et al. 2011, AJ, 141, 175

Székely, P., Kiss, L. L., Jackson, R., et al. 2007, A\&A, 463, 589

Thygesen, A. O., Sbordone, L., Andrievsky, S., et al. 2014, A\&A, 572, A108

Ting, Y. S., Conroy, C., Rix, H. W., \& Cargile, P. 2019, ApJ, 879, 69

Turri, P., McConnachie, A. W., Stetson, P. B., et al. 2015, ApJ, 811, L15

VandenBerg, D. A., Bergbusch, P. A., Ferguson, J. W., \& Edvardsson, B. 2014, ApJ, 794, 72

Villanova, S., \& Geisler, D. 2011, A\&A, 535, A31

Wagner-Kaiser, R., Sarajedini, A., von Hippel, T., et al. 2017, MNRAS, 468, 1038

Walker, M. G., Mateo, M., Olszewski, E. W., et al. 2006, AJ, 131, 2114

Worley, C. C., \& Cottrell, P. L. 2010, MNRAS, 406, 2504

Worley, C. C., Hill, V., Sobeck, J., \& Carretta, E. 2013, A\&A, 553, A47

Yakovleva, S. A., Voronov, Y. V., \& Belyaev, A. K. 2016, A\&A, 593, A27

Yong, D., \& Grundahl, F. 2008, ApJ, 672, L29

Yong, D., Grundahl, F., Nissen, P. E., Jensen, H. R., \& Lambert, D. L. 2005 A\&A, 438, 875

Yong, D., Meléndez, J., Grundahl, F., et al. 2013, MNRAS, 434, 3542

Yong, D., Roederer, I. U., Grundahl, F., et al. 2014, MNRAS, 441, 3396

Yong, D., Grundahl, F., \& Norris, J. E. 2015, MNRAS, 446, 3319

Zhao, G., Mashonkina, L., Yan, H. L., et al. 2016, ApJ, 833, 225 


\section{Appendix A: Supplementary tables}

In Table A.1 we list the sensitivity of measured abundance ratios to the typical errors in atmospheric parameters. We run the analysis with one parameter fixed to a perturbed value, allowing code to fit the others. We list the quadratic sum of individual sensitivities in the last row as the total systematic error. The results are given for one star from the open cluster NGC 2243, two stars in the metal-rich cluster NGC 104 and two stars in metal-poor cluster M 15.

In Table A.2, we provide NLTE stellar parameters for the highest S/N spectra of 19 Gaia benchmark stars along with reference values from Jofré et al. (2015). The last row indicates the mean difference of our values.

In Table A.3, we list cluster parameters from the literature including equatorial coordinates, heliocentric distances, reddening, mean $\mathrm{RV}$, age and $[\mathrm{Fe} / \mathrm{H}]$.

In Table A.4, we provide maximum likelihood estimates of the cluster average abundances and internal dispersions (more precisely, abundance dispersion of the cluster that is not accounted for by the abundance measurement systematic error) with associated errors.

In Table A.5, we provide NLTE/LTE stellar parameters and abundances with systematic errors for all 742 stars in the cluster sample.
Table A.1. Sensitivity of abundance ratios to errors in atmospheric parameters.

\begin{tabular}{|c|c|c|c|}
\hline Star/parameter & $\begin{array}{c}\Delta[\mathrm{Fe} / \mathrm{H}] \\
\operatorname{dex}\end{array}$ & $\begin{array}{c}\Delta[\mathrm{Mg} / \mathrm{Fe}] \\
\operatorname{dex}\end{array}$ & $\begin{array}{c}\Delta[\mathrm{Ti} / \mathrm{Fe}] \\
\operatorname{dex}\end{array}$ \\
\hline \multicolumn{4}{|c|}{$06291929-3125331 T_{\text {eff }}=6689, \log (g)=4.22,[\mathrm{Fe} / \mathrm{H}]=-0.52$} \\
\hline$T_{\text {eff }}+150 \mathrm{~K}$ & 0.08 & -0.01 & 0.01 \\
\hline $\log (g)+0.3 \mathrm{dex}$ & 0.07 & -0.06 & 0.08 \\
\hline$[\mathrm{Fe} / \mathrm{H}]+0.1 \mathrm{dex}$ & & -0.02 & 0.01 \\
\hline$V_{\text {mic }}+0.2 \mathrm{~km} \mathrm{~s}^{-1}$ & 0.01 & 0.02 & -0.01 \\
\hline Total & 0.10 & 0.06 & 0.07 \\
\hline \multicolumn{4}{|c|}{$00225472-7203461 T_{\text {eff }}=5146, \log (g)=3.08,[\mathrm{Fe} / \mathrm{H}]=-0.75$} \\
\hline$T_{\text {eff }}+150 \mathrm{~K}$ & 0.11 & -0.07 & 0.04 \\
\hline $\log (g)+0.3 \mathrm{dex}$ & 0.08 & -0.10 & 0.08 \\
\hline$[\mathrm{Fe} / \mathrm{H}]+0.1 \mathrm{dex}$ & & -0.05 & 0.02 \\
\hline$V_{\text {mic }}+0.2 \mathrm{~km} \mathrm{~s}^{-1}$ & -0.03 & 0.02 & -0.04 \\
\hline Total & 0.14 & 0.14 & 0.10 \\
\hline \multicolumn{4}{|c|}{$00250332-7201108 T_{\text {eff }}=4662, \log (g)=2.21,[\mathrm{Fe} / \mathrm{H}]=-0.78$} \\
\hline$T_{\text {eff }}+150 \mathrm{~K}$ & 0.13 & -0.08 & 0.03 \\
\hline $\log (g)+0.3 \mathrm{dex}$ & 0.08 & -0.09 & 0.04 \\
\hline$[\mathrm{Fe} / \mathrm{H}]+0.1 \mathrm{dex}$ & $\ldots$ & -0.04 & -0.01 \\
\hline$V_{\mathrm{mic}}+0.2 \mathrm{~km} \mathrm{~s}^{-1}$ & -0.04 & 0.02 & -0.02 \\
\hline Total & 0.16 & 0.12 & 0.05 \\
\hline \multicolumn{4}{|c|}{$21300747+1210115 T_{\text {eff }}=5150, \log (g)=1.99,[\mathrm{Fe} / \mathrm{H}]=-2.32$} \\
\hline$T_{\text {eff }}+150 \mathrm{~K}$ & 0.10 & -0.05 & -0.01 \\
\hline $\log (g)+0.3 \mathrm{dex}$ & 0.01 & -0.01 & 0.10 \\
\hline$[\mathrm{Fe} / \mathrm{H}]+0.1 \mathrm{dex}$ & $\ldots$ & -0.05 & -0.03 \\
\hline$V_{\text {mic }}+0.2 \mathrm{~km} \mathrm{~s}^{-1}$ & -0.04 & 0.02 & -0.01 \\
\hline Total & 0.11 & 0.08 & 0.11 \\
\hline \multicolumn{4}{|c|}{$21295615+1210296 T_{\text {eff }}=5329, \log (g)=2.30,[\mathrm{Fe} / \mathrm{H}]=-2.26$} \\
\hline$T_{\text {eff }}+150 \mathrm{~K}$ & 0.10 & -0.02 & 0.02 \\
\hline $\log (g)+0.3 \mathrm{dex}$ & 0.06 & -0.01 & 0.06 \\
\hline$[\mathrm{Fe} / \mathrm{H}]+0.1 \mathrm{dex}$ & $\ldots$ & -0.03 & 0.01 \\
\hline$V_{\text {mic }}+0.2 \mathrm{~km} \mathrm{~s}^{-1}$ & 0.06 & -0.01 & 0.03 \\
\hline Total & 0.13 & 0.03 & 0.07 \\
\hline
\end{tabular}


Table A.2. Gaia benchmark stars parameters from NLTE fit ( $\max \mathrm{S} / \mathrm{N}$ ) and reference study (Jofré et al. 2015), except where noted.

\begin{tabular}{|c|c|c|c|c|}
\hline Star & $\begin{array}{l}T_{\text {eff }}, \mathrm{K} \\
\text { fit, ref }\end{array}$ & $\begin{array}{l}\log (g), \operatorname{dex} \\
\text { fit, ref }\end{array}$ & $\begin{array}{c}{[\mathrm{Fe} / \mathrm{H}], \mathrm{dex}} \\
\text { fit, ref }\end{array}$ & $\begin{array}{l}V_{\text {mic }}, \mathrm{km} \mathrm{s}^{-1} \\
\text { fit, ref }\end{array}$ \\
\hline HD 107328 & $4384,4496 \pm 59$ & $1.90,2.09 \pm 0.14$ & $-0.60,-0.38 \pm 0.16$ & $1.71,1.65 \pm 0.26$ \\
\hline HD 220009 & $4336,4275 \pm 54$ & $1.86,1.47 \pm 0.14$ & $-0.79,-0.79 \pm 0.13$ & $1.42,1.49 \pm 0.14$ \\
\hline ksiHya & $5045,5044 \pm 38$ & $3.01,2.87 \pm 0.02$ & $-0.05,0.11 \pm 0.20$ & $1.54,1.40 \pm 0.32$ \\
\hline muLeo & $4462,4474 \pm 60$ & $2.45,2.51 \pm 0.09$ & $0.01,0.20 \pm 0.15$ & $1.54,1.28 \pm 0.26$ \\
\hline HD 122563 & $4771,4636 \pm 37^{(1)}$ & $1.29,1.42 \pm 0.01^{(2)}$ & $-2.56,-2.52 \pm 0.11^{(3)}$ & $2.53,1.92 \pm 0.11$ \\
\hline HD 140283 & $5888,5787 \pm 48^{(1)}$ & $3.63,3.57 \pm 0.12$ & $-2.39,-2.34 \pm 0.03^{(3)}$ & $2.16,1.56 \pm 0.20$ \\
\hline delEri & $5006,4954 \pm 26$ & $3.61,3.75 \pm 0.02$ & $-0.00,0.01 \pm 0.05$ & $1.15,1.10 \pm 0.22$ \\
\hline epsFor & $5070,5123 \pm 78$ & $3.28,3.52 \pm 0.07$ & $-0.65,-0.65 \pm 0.10$ & $1.14,1.04 \pm 0.13$ \\
\hline $18 \mathrm{Sco}$ & $5838,5810 \pm 80$ & $4.32,4.44 \pm 0.03$ & $0.02,-0.02 \pm 0.03$ & $1.27,1.07 \pm 0.20$ \\
\hline alfCenB & $5167,5231 \pm 20$ & $4.33,4.53 \pm 0.03$ & $0.14,0.17 \pm 0.10$ & $1.06,0.99 \pm 0.31$ \\
\hline muAra & $5743,5902 \pm 66$ & $4.05,4.30 \pm 0.03$ & $0.22,0.30 \pm 0.13$ & $1.32,1.17 \pm 0.13$ \\
\hline betVir & $6259,6083 \pm 41$ & $4.06,4.10 \pm 0.02$ & $0.18,0.19 \pm 0.07$ & $1.51,1.33 \pm 0.09$ \\
\hline epsEri & $5079,5076 \pm 30$ & $4.54,4.60 \pm 0.03$ & $-0.14,-0.14 \pm 0.06$ & $1.11,1.14 \pm 0.05$ \\
\hline etaBoo & $6183,6099 \pm 28$ & $3.84,3.80 \pm 0.02$ & $0.27,0.27 \pm 0.08$ & $1.52,1.52 \pm 0.19$ \\
\hline HD 22879 & $5907,5868 \pm 89$ & $3.98,4.27 \pm 0.03$ & $-0.80,-0.91 \pm 0.05$ & $1.24,1.05 \pm 0.19$ \\
\hline HD 49933 & $6718,6635 \pm 91$ & $4.16,4.20 \pm 0.03$ & $-0.36,-0.46 \pm 0.08$ & $1.51,1.46 \pm 0.35$ \\
\hline HD 84937 & $6481,6356 \pm 97$ & $3.91,4.15 \pm 0.06$ & $-2.00,-1.99 \pm 0.02^{(3)}$ & $1.76,1.39 \pm 0.24$ \\
\hline Procyon & $6686,6554 \pm 84$ & $3.91,3.99 \pm 0.02$ & $0.03,-0.04 \pm 0.08$ & $1.83,1.66 \pm 0.11$ \\
\hline tauCet & $5349,5414 \pm 21$ & $4.26,4.49 \pm 0.01$ & $-0.52,-0.54 \pm 0.03$ & $1.00,0.89 \pm 0.28$ \\
\hline$\langle$ ref-fit $\rangle$ & $-29 \pm 88$ & $0.09 \pm 0.16$ & $0.02 \pm 0.09$ & $-0.16 \pm 0.18$ \\
\hline
\end{tabular}

Notes. In order to be consistent with our reference solar $[\mathrm{Fe} / \mathrm{H}]$ scale, we subtracted 0.05 dex from Jofré et al. (2015) and 0.03 dex from Amarsi et al. (2016) metallicities.

References. ${ }^{(1)}$ Karovicova et al. (2018). ${ }^{(2)}$ Creevey et al. (2019). ${ }^{(3)}$ Amarsi et al. (2016).

Table A.3. Cluster parameters used in this work.

\begin{tabular}{lccccccc}
\hline \hline Cluster & $\alpha, \mathrm{deg}$ & $\delta, \mathrm{deg}$ & $d_{\odot}, \mathrm{kpc}$ & $E(B-V)$ & $\langle\mathrm{RV}\rangle, \mathrm{km} \mathrm{s}^{-1}$ & Age, Gyr & {$[\mathrm{Fe} / \mathrm{H}], \mathrm{dex}$} \\
\hline NGC 3532 (oc) & 166.4125 & -58.7533 & $0.5(1)$ & $0.03(1)$ & 4.3 & 0.3 & -0.01 \\
NGC 5927 (gc) & 232.0029 & -50.6730 & 7.7 & 0.45 & -100.5 & 11.9 & -0.48 \\
NGC 2243 (oc) & 97.3917 & -31.2833 & 4.5 & 0.05 & 59.8 & $3.8^{(2)}$ & $-0.57^{(2)}$ \\
NGC 104 (gc) & 6.0224 & -72.0815 & $4.5^{(3)}$ & 0.04 & -18.7 & 12.5 & -0.75 \\
NGC 1851 (gc) & 78.5281 & -40.0465 & 12.1 & 0.02 & 320.9 & 10.5 & -1.10 \\
NGC 2808 (gc) & 138.0129 & -64.8635 & 9.6 & 0.22 & 102.8 & 10.9 & -1.14 \\
NGC 362 (gc) & 15.8094 & -70.8488 & $8.5(3)$ & 0.05 & 222.9 & 10.9 & -1.23 \\
M 2 (gc) & 323.3626 & -0.8233 & 11.5 & 0.06 & -6.7 & 12.0 & -1.52 \\
NGC 6752 (gc) & 287.7170 & -59.9846 & 4.0 & 0.02 & -27.4 & 12.3 & -1.43 \\
NGC 1904 (gc) & 81.0441 & -24.5242 & 12.9 & 0.01 & 205.8 & 11.1 & -1.37 \\
NGC 4833 (gc) & 194.8913 & -70.8765 & 6.6 & 0.32 & 201.1 & 12.7 & -1.97 \\
NGC 4372 (gc) & 186.4393 & -72.6591 & 5.8 & $0.30 . .0 .80$ & 72.6 & 12.5 & -1.88 \\
M 15 (gc) & 322.4930 & 12.1670 & 10.4 & 0.10 & -106.6 & 13.0 & -2.25 \\
\hline
\end{tabular}

Notes. The coordinates and radial velocities from SIMBAD database, ages and [Fe/H] from Kruijssen et al. (2019) for globular clusters (gc) and WEBDA database for open clusters (oc), distances and $E(B-V)$ are from Harris (1996, 2010 edition) (gc) or WEBDA (oc) databases, except where noted.

References. ${ }^{(1)}$ Fritzewski et al. (2019). ${ }^{(2)}$ Anthony-Twarog et al. (2005). ${ }^{(3)}$ Chen et al. (2018). ${ }^{(4)}$ Kacharov et al. (2014). 
Table A.4. Maximum likelihood estimates of the cluster average abundances and internal dispersions.

\begin{tabular}{|c|c|c|c|c|c|c|}
\hline $\begin{array}{l}\text { Cluster } \\
N \text { stars }\end{array}$ & $\begin{array}{c}\left\langle[\mathrm{Fe} / \mathrm{H}]_{\text {NLTE }}\right\rangle \\
\left\langle[\mathrm{Fe} / \mathrm{H}]_{\text {LTE }}\right\rangle \\
\operatorname{dex}\end{array}$ & $\begin{array}{c}\sigma[\mathrm{Fe} / \mathrm{H}]_{\mathrm{NLTE}} \\
\sigma[\mathrm{Fe} / \mathrm{H}]_{\mathrm{LTE}} \\
\operatorname{dex}\end{array}$ & $\begin{array}{c}\left\langle[\mathrm{Mg} / \mathrm{Fe}]_{\text {NLTE }}\right\rangle \\
\left\langle[\mathrm{Mg} / \mathrm{Fe}]_{\text {LTE }}\right\rangle \\
\operatorname{dex}\end{array}$ & $\begin{array}{c}\sigma[\mathrm{Mg} / \mathrm{Fe}]_{\mathrm{NLTE}} \\
\sigma[\mathrm{Mg} / \mathrm{Fe}]_{\mathrm{LTE}} \\
\operatorname{dex}\end{array}$ & $\begin{array}{c}\left\langle[\mathrm{Ti} / \mathrm{Fe}]_{\mathrm{NLTE}}\right\rangle \\
\left\langle[\mathrm{Ti} / \mathrm{Fe}]_{\mathrm{LTE}}\right\rangle \\
\operatorname{dex}\end{array}$ & $\begin{array}{c}\sigma[\mathrm{Ti} / \mathrm{Fe}]_{\mathrm{NLTE}} \\
\sigma[\mathrm{Ti} / \mathrm{Fe}]_{\mathrm{LTE}} \\
\operatorname{dex}\end{array}$ \\
\hline NGC 3532 & $-0.10 \pm 0.02$ & $0.00 \pm 0.02$ & $-0.09 \pm 0.03$ & $0.00 \pm 0.03$ & $0.01 \pm 0.03$ & $0.00 \pm 0.03$ \\
\hline 12 & $-0.09 \pm 0.02$ & $0.00 \pm 0.03$ & $-0.07 \pm 0.03$ & $0.00 \pm 0.03$ & $0.01 \pm 0.03$ & $0.00 \pm 0.03$ \\
\hline NGC 5927 & $-0.48 \pm 0.02$ & $0.00 \pm 0.02$ & $0.39 \pm 0.02$ & $0.00 \pm 0.02$ & $0.29 \pm 0.01$ & $0.00 \pm 0.02$ \\
\hline 47 & $-0.49 \pm 0.02$ & $0.00 \pm 0.02$ & $0.41 \pm 0.02$ & $0.00 \pm 0.02$ & $0.23 \pm 0.01$ & $0.00 \pm 0.02$ \\
\hline NGC 2243 & $-0.52 \pm 0.01$ & $0.00 \pm 0.01$ & $0.15 \pm 0.01$ & $0.00 \pm 0.02$ & $0.02 \pm 0.01$ & $0.00 \pm 0.04$ \\
\hline 84 & $-0.57 \pm 0.01$ & $0.00 \pm 0.02$ & $0.26 \pm 0.01$ & $0.00 \pm 0.02$ & $0.01 \pm 0.01$ & $0.00 \pm 0.05$ \\
\hline NGC 104 & $-0.74 \pm 0.02$ & $0.00 \pm 0.02$ & $0.38 \pm 0.02$ & $0.00 \pm 0.02$ & $0.30 \pm 0.01$ & $0.00 \pm 0.02$ \\
\hline 68 & $-0.75 \pm 0.02$ & $0.00 \pm 0.02$ & $0.42 \pm 0.02$ & $0.00 \pm 0.02$ & $0.26 \pm 0.01$ & $0.00 \pm 0.03$ \\
\hline NGC 1851 & $-1.11 \pm 0.01$ & $0.00 \pm 0.02$ & $0.22 \pm 0.01$ & $0.00 \pm 0.02$ & $0.28 \pm 0.01$ & $0.00 \pm 0.02$ \\
\hline 88 & $-1.15 \pm 0.01$ & $0.00 \pm 0.02$ & $0.36 \pm 0.01$ & $0.00 \pm 0.02$ & $0.24 \pm 0.01$ & $0.00 \pm 0.01$ \\
\hline NGC 2808 & $-1.01 \pm 0.03$ & $0.00 \pm 0.03$ & $0.11 \pm 0.03$ & $0.09 \pm 0.03$ & $0.33 \pm 0.02$ & $0.00 \pm 0.02$ \\
\hline 25 & $-1.03 \pm 0.03$ & $0.00 \pm 0.03$ & $0.22 \pm 0.03$ & $0.00 \pm 0.09$ & $0.30 \pm 0.01$ & $0.00 \pm 0.02$ \\
\hline NGC 362 & $-1.05 \pm 0.02$ & $0.00 \pm 0.02$ & $0.15 \pm 0.01$ & $0.00 \pm 0.02$ & $0.29 \pm 0.01$ & $0.00 \pm 0.01$ \\
\hline 62 & $-1.09 \pm 0.02$ & $0.00 \pm 0.02$ & $0.26 \pm 0.02$ & $0.00 \pm 0.02$ & $0.26 \pm 0.01$ & $0.00 \pm 0.01$ \\
\hline M 2 & $-1.47 \pm 0.01$ & $0.00 \pm 0.02$ & $0.17 \pm 0.01$ & $0.07 \pm 0.01$ & $0.23 \pm 0.01$ & $0.00 \pm 0.02$ \\
\hline 78 & $-1.54 \pm 0.01$ & $0.00 \pm 0.02$ & $0.34 \pm 0.02$ & $0.08 \pm 0.02$ & $0.25 \pm 0.01$ & $0.00 \pm 0.01$ \\
\hline NGC 6752 & $-1.48 \pm 0.01$ & $0.00 \pm 0.01$ & $0.20 \pm 0.01$ & $0.03 \pm 0.02$ & $0.17 \pm 0.01$ & $0.00 \pm 0.01$ \\
\hline 110 & $-1.56 \pm 0.01$ & $0.00 \pm 0.01$ & $0.35 \pm 0.01$ & $0.04 \pm 0.02$ & $0.23 \pm 0.01$ & $0.00 \pm 0.01$ \\
\hline NGC 1904 & $-1.51 \pm 0.02$ & $0.00 \pm 0.02$ & $0.16 \pm 0.01$ & $0.04 \pm 0.02$ & $0.21 \pm 0.01$ & $0.00 \pm 0.02$ \\
\hline 44 & $-1.60 \pm 0.02$ & $0.00 \pm 0.02$ & $0.31 \pm 0.02$ & $0.00 \pm 0.04$ & $0.24 \pm 0.01$ & $0.00 \pm 0.03$ \\
\hline NGC 4833 & $-1.88 \pm 0.02$ & $0.00 \pm 0.02$ & $0.18 \pm 0.03$ & $0.15 \pm 0.02$ & $0.22 \pm 0.01$ & $0.00 \pm 0.02$ \\
\hline 33 & $-2.08 \pm 0.02$ & $0.00 \pm 0.03$ & $0.36 \pm 0.03$ & $0.18 \pm 0.03$ & $0.24 \pm 0.02$ & $0.00 \pm 0.02$ \\
\hline NGC 4372 & $-2.07 \pm 0.02$ & $0.00 \pm 0.02$ & $0.31 \pm 0.01$ & $0.00 \pm 0.03$ & $0.20 \pm 0.01$ & $0.00 \pm 0.02$ \\
\hline 45 & $-2.33 \pm 0.02$ & $0.00 \pm 0.02$ & $0.51 \pm 0.02$ & $0.00 \pm 0.04$ & $0.22 \pm 0.01$ & $0.00 \pm 0.02$ \\
\hline M 15 & $-2.28 \pm 0.02$ & $0.00 \pm 0.02$ & $0.22 \pm 0.03$ & $0.16 \pm 0.02$ & $0.21 \pm 0.02$ & $0.00 \pm 0.02$ \\
\hline 46 & $-2.58 \pm 0.02$ & $0.00 \pm 0.02$ & $0.36 \pm 0.04$ & $0.22 \pm 0.03$ & $0.19 \pm 0.01$ & $0.00 \pm 0.02$ \\
\hline
\end{tabular}

Table A.5. NLTE and LTE stellar parameters, abundance ratios with uncertainties for the 742 stars in clusters sample.

\begin{tabular}{|c|c|c|c|c|c|c|c|c|c|c|c|c|}
\hline \multirow{2}{*}{$\begin{array}{l}\text { Star } \\
\text { HHMMSSss-DDMMSSs } \\
\end{array}$} & \multirow[t]{2}{*}{ Cluster } & \multirow{2}{*}{$\begin{array}{l}S / N \\
\AA^{-1} \\
\end{array}$} & \multicolumn{2}{|c|}{$T_{\text {eff }}$} & \multicolumn{2}{|c|}{$\log (g)$} & \multicolumn{2}{|c|}{$V_{\text {mic }}$} & \multicolumn{2}{|c|}{$[\mathrm{Fe} / \mathrm{H}]$} & \multicolumn{2}{|c|}{$\Delta[\mathrm{Fe} / \mathrm{H}]$} \\
\hline & & & $\begin{array}{c}\text { NLTE } \\
\mathrm{K}\end{array}$ & $\begin{array}{c}\text { LTE } \\
\mathrm{K}\end{array}$ & $\begin{array}{c}\text { NLTE } \\
\text { dex }\end{array}$ & $\begin{array}{l}\text { LTE } \\
\text { dex }\end{array}$ & $\begin{array}{l}\text { NLTE } \\
\mathrm{km} \mathrm{s}^{-1}\end{array}$ & $\begin{array}{c}\mathrm{LTE} \\
\mathrm{km} \mathrm{s}^{-1}\end{array}$ & $\begin{array}{l}\text { NLTE } \\
\text { dex }\end{array}$ & $\begin{array}{l}\text { LTE } \\
\text { dex }\end{array}$ & $\begin{array}{l}\text { NLTE } \\
\text { dex }\end{array}$ & $\begin{array}{l}\text { LTE } \\
\text { dex }\end{array}$ \\
\hline $11070908-5839114$ & NGC 3532 & 537.5 & 5389 & 5379 & 4.53 & 4.52 & 1.39 & 1.28 & -0.10 & -0.09 & 0.09 & 0.08 \\
\hline $11071871-5849027$ & NGC 3532 & 521.9 & 5606 & 5615 & 4.54 & 4.54 & 1.43 & 1.41 & -0.08 & -0.08 & 0.09 & 0.09 \\
\hline \multirow[t]{5}{*}{$\ldots$} & \multicolumn{2}{|c|}{ ‥ $\mathrm{Mg} / \mathrm{Fe}]$} & \multicolumn{2}{|c|}{$\begin{array}{lr}\ldots & \ldots \\
\Delta[\mathrm{Mg} / \mathrm{Fe}] \\
\end{array}$} & \multicolumn{2}{|c|}{$\begin{array}{cc}\ldots & \ldots \\
& {[\mathrm{Ti} / \mathrm{Fe}]} \\
\end{array}$} & \multicolumn{2}{|c|}{$\begin{array}{cc}\ldots & \ldots \\
\Delta[\mathrm{Ti} / \mathrm{Fe}]\end{array}$} & \multicolumn{2}{|c|}{$\begin{array}{cc}\ldots & \ldots \\
& {[\mathrm{Mn} / \mathrm{Fe}]} \\
\end{array}$} & \multicolumn{2}{|c|}{$\begin{array}{ll}\ldots & \ldots \\
\Delta[\mathrm{Mn} / \mathrm{Fe}] \\
\end{array}$} \\
\hline & $\begin{array}{l}\text { NLTE } \\
\operatorname{dex}\end{array}$ & $\begin{array}{c}\text { LTE } \\
\text { dex }\end{array}$ & $\begin{array}{l}\text { NLTE } \\
\text { dex }\end{array}$ & $\begin{array}{l}\text { LTE } \\
\text { dex }\end{array}$ & $\begin{array}{l}\text { NLTE } \\
\text { dex }\end{array}$ & $\begin{array}{l}\text { LTE } \\
\text { dex }\end{array}$ & $\begin{array}{l}\text { NLTE } \\
\text { dex }\end{array}$ & $\begin{array}{l}\text { LTE } \\
\text { dex }\end{array}$ & $\begin{array}{l}\text { NLTE } \\
\text { dex }\end{array}$ & $\begin{array}{l}\text { LTE } \\
\text { dex }\end{array}$ & $\begin{array}{l}\text { NLTE } \\
\text { dex }\end{array}$ & $\begin{array}{l}\text { LTE } \\
\text { dex }\end{array}$ \\
\hline & -0.08 & -0.08 & 0.09 & 0.10 & -0.01 & 0.04 & 0.08 & 0.14 & -0.14 & -0.26 & 0.11 & 0.13 \\
\hline & -0.08 & -0.07 & 0.09 & 0.10 & 0.02 & 0.03 & 0.06 & 0.10 & -0.16 & -0.26 & 0.12 & 0.12 \\
\hline & $\ldots$ & $\ldots$ & $\ldots$ & $\ldots$ & $\ldots$ & $\ldots$ & $\ldots$ & $\ldots$ & $\ldots$ & $\ldots$ & $\ldots$ & $\cdots$ \\
\hline
\end{tabular}

Notes. The full table is available at the CDS. 


\section{Appendix B: Instrumental profile}

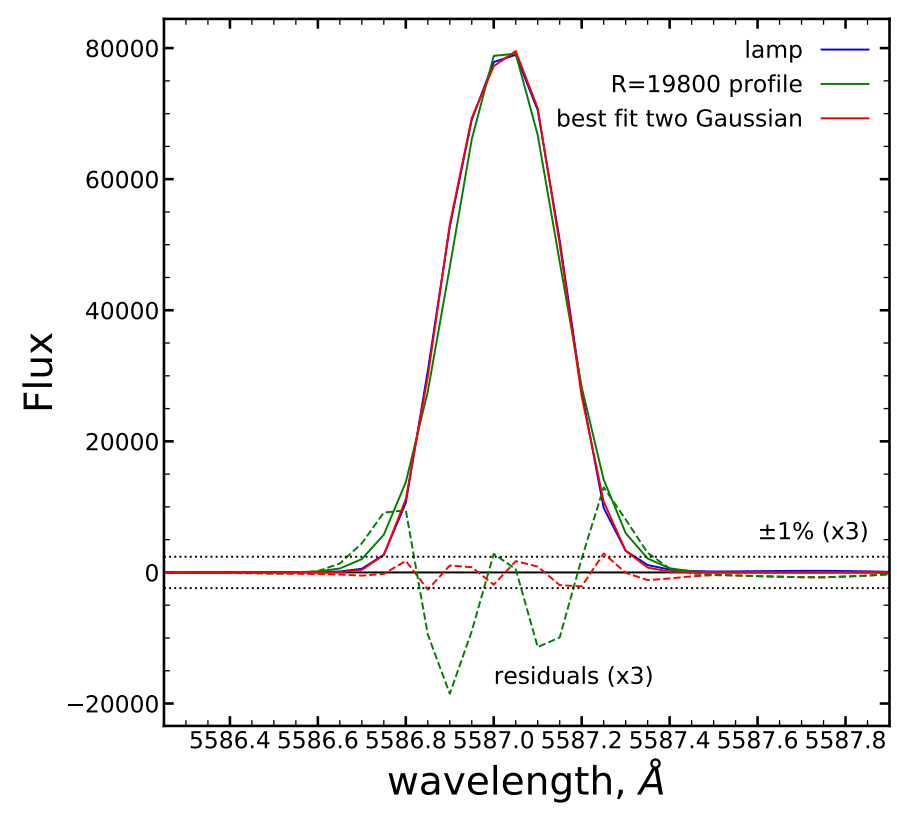

Fig. B.1. Results of instrumental profile fitting. Residuals of the fit are up-scaled three times.

Similarly to the technique that Damiani et al. (2016) used to obtain an instrumental profile for the Giraffe HR15N setting, we used a sum of two Gaussian profiles to fit the line at $5578 \AA$ in the calibration spectrum of the thorium-argon lamp, downloaded from the ESO webpage ${ }^{13}$. It is shown in Fig. B.1 that such a new instrumental profile describes the spectral profile much better than a single Gaussian computed according to the reported resolution of HR10 setting $R=19800$. The error of a single Gaussian profile can be up to 5-7

$$
\lambda(v)=\frac{A_{1}}{\sqrt{2 \pi \sigma_{1}^{2}}} \exp \left(-\frac{\left(v-v_{1}\right)^{2}}{2 \sigma_{1}^{2}}\right)+\frac{A_{2}}{\sqrt{2 \pi \sigma_{2}^{2}}} \exp \left(-\frac{\left(v-v_{2}\right)^{2}}{2 \sigma_{2}^{2}}\right)
$$

with $A_{1}=0.465, A_{2}=0.194, \sigma_{1}=4.971 \mathrm{~km} \mathrm{~s}^{-1}, \sigma_{2}=$ $3.799 \mathrm{~km} \mathrm{~s}^{-1}, v_{1}=-2.249 \mathrm{~km} \mathrm{~s}^{-1}, v_{2}=5.754 \mathrm{~km} \mathrm{~s}^{-1}$.

\section{Appendix C: Kinematic assignment of the populations.}

We employed the cluster distances listed in Table A.3. They were obtained from the colour magnitude diagram horizontal branch (globular clusters Harris (1996, 2010 edition)) or turn-off point (open clusters WEBDA database) fitting. The same distance was assumed for all stars within given cluster. We also take proper motions from Gaia DR2 (Gaia Collaboration 2018) and radial velocities from our analysis and compute galactocentric rectangular velocity components $(\mathrm{U}, \mathrm{V}, \mathrm{W})$ for all stars in the clusters, using Astropy (Astropy Collaboration 2013), package, with respect to solar motion from Schönrich et al. (2010).

The computed velocities were used to calculate the probability ratios $T D / D$ and $T D / H$ (Bensby et al. 2014, Appendix 1), which allowed us to assign population membership to the clusters. We used the following selection criteria: thick disk if $T D / D>2$ and $T D / H>1$; thin disk if $T D / D<0.5$; halo if $T D / H<1$. Only the open cluster NGC 2243 has a probability ratio of $T D / D=1.25$ in between the thin and the thick disk. We therefore decided to assign it to the thick disk on the basis of its large separation $(|z|=1 \mathrm{kpc})$ from the Galactic plane.

\footnotetext{
13 http://wWw . eso.org/observing/dfo/quality/GIRAFFE/ pipeline/SKY/html/GI_SRBS_2004-09-26T22_48_10.511_ Medusa2_H548.8nm_o10.fits_details.html
} 